\title{
Improved calculations of the complex dielectric constant of semiconductors
}

\author{
Roger Allen Breckenridge \\ College of William \& Mary - Arts \& Sciences
}

Follow this and additional works at: https://scholarworks.wm.edu/etd

Part of the Condensed Matter Physics Commons

\section{Recommended Citation}

Breckenridge, Roger Allen, "Improved calculations of the complex dielectric constant of semiconductors" (1974). Dissertations, Theses, and Masters Projects. Paper 1539623675.

https://dx.doi.org/doi:10.21220/s2-2anm-4k02

This Dissertation is brought to you for free and open access by the Theses, Dissertations, \& Master Projects at W\&M ScholarWorks. It has been accepted for inclusion in Dissertations, Theses, and Masters Projects by an authorized administrator of W\&M ScholarWorks. For more information, please contact scholarworks@wm.edu. 


\section{INFORMATION TO USERS}

This material was produced from a microfilm copy of the original document. While the most advanced technological means to photograph and reproduce this document have been used, the quality is heavily dependent upon the quality of the original submittod.

The following explanation of techniques is provided to help you understand markings or patterns which may appear on this reproduction.

1. The sign or "target" for pages apparently lacking from the document photographed is "Missing Page(s)". If it was possible to obtain the missing page(s) or section, they are spliced into the film along with adjacent pages. This may have necessitated cutting thru an image and duplicating adjacent pages to insure you complete continuity.

2. When an image on the film is obliterated with a large round black mark, it is an indication that the photographer suspected that the copy may have moved during exposure and thus cause a blurred image. You will find a good image of the page in the adjacent frame.

3. When a map, drawing or chart, etc., was part of the material being photographed the photographer followed a definite method in "sectioning" the material. It is customary to begin photoing at the upper left hand corner of a large sheet and to continue photoing from left to right in equal sections with a small overlap. If necessary, sectioning is continued again - beginning below the first row and continuing on until complete.

4. The majority of users indicate that the textual content is of greatest value, however, a somewhat higher quality reproduction could be made from "photographs" if essential to the understanding of the dissertation. Silver prints of "photographs" may be ordered at additional charge by writing the Order Department, giving the catalog number, title, author and specific pages you wish reproduced.

5. PLEASE NOTE: Some pages may have indistinct print. Filmed as received.

Xerox University Microfilms 


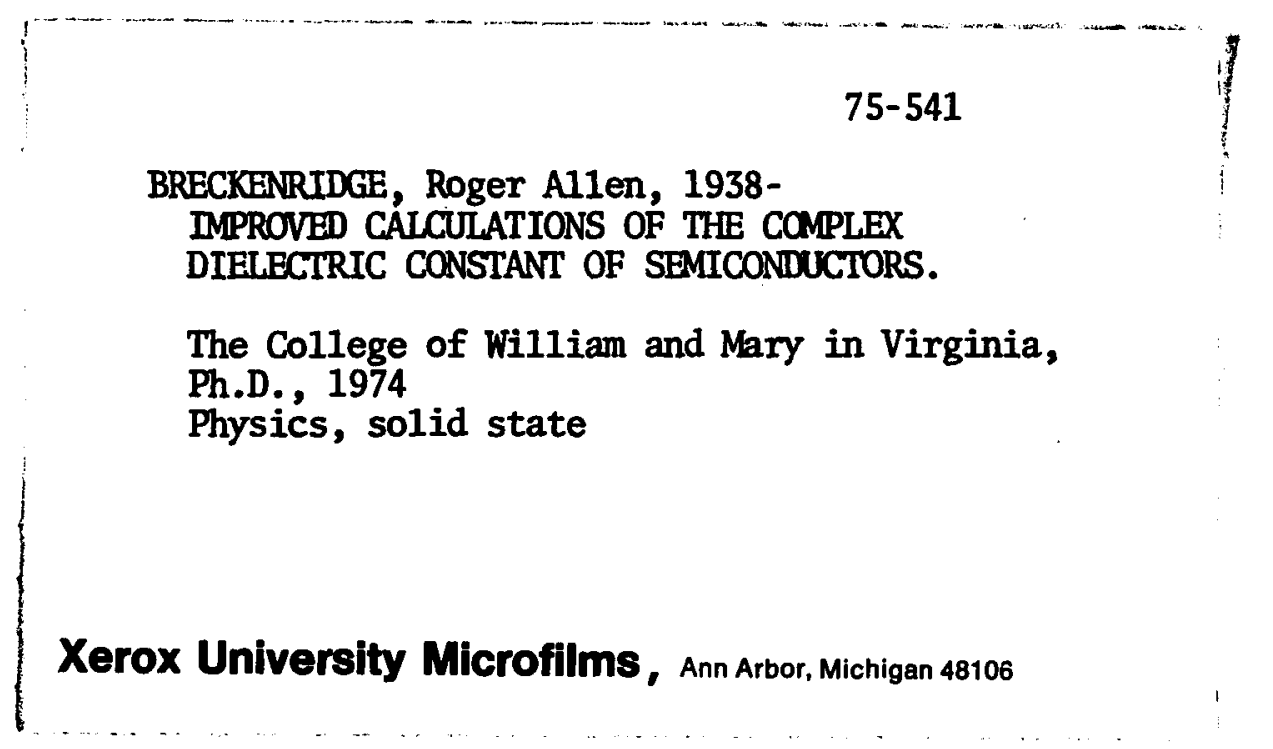


IMPROVED CALCULATIONS OF THE COMPLEX

DIELECTRIC CONSTANT OF SEMICONDUCTORS

\author{
A Thesis \\ Presented to \\ The Faculty of the Department of Physics \\ The College of William and Mary in Virginia
}

In Partial Fulfillment

Of the Requirements for the Degree of

Doctor of Philosophy

by

Roger A. Breckenridge

August 1974 


\section{L.D. $D$}

APPROVAL SHEET

This dissertation is submitted in partial fulfillment of the requirements for the degree of

Doctor of Philosophy

Approved, July 1974
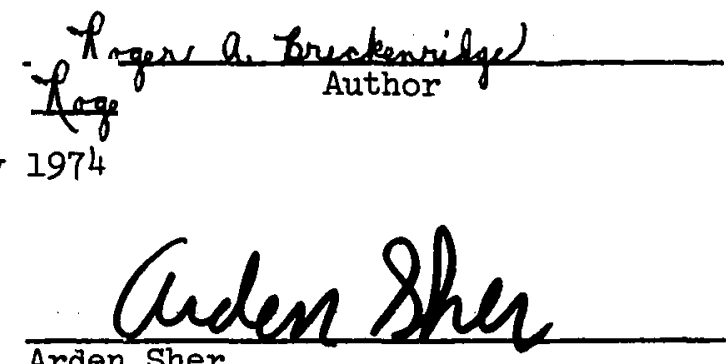

Arden Sher
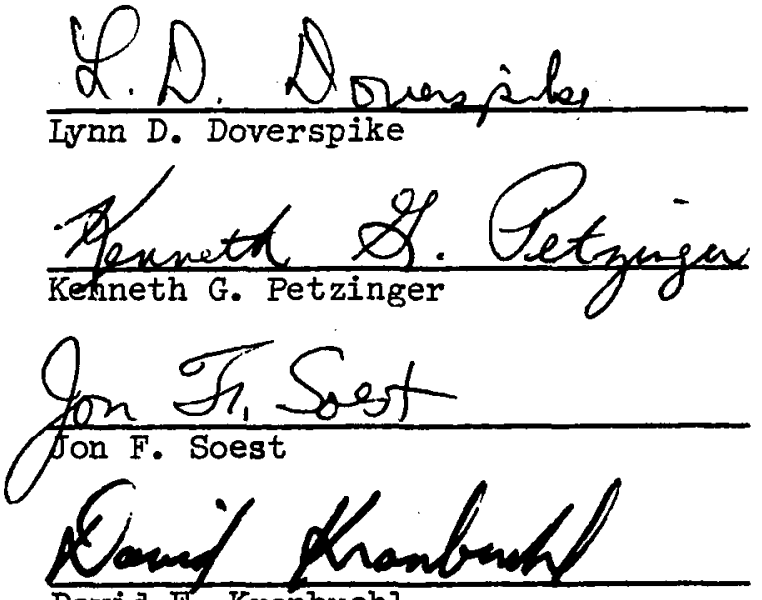

David E. Kranbuehl

Department of Chemistry 
IMPROVED CALCULATIONS OF THE COMPLEX

DIELECTRIC CONSTANT OF SEMICONDUCTORS 
TABLE OF CONTENTS

I. INTRODUCTION. . . . . . . . . . . . . . I

II. THE SELF-CONSISTENT-FIELD METHOD FOR CALCULATION

of $\in(\omega, q) \ldots \ldots \ldots \ldots$

III. CALCULATION OF $\epsilon_{1}(0)$ AND $\epsilon_{2}(\omega)$ USING THE

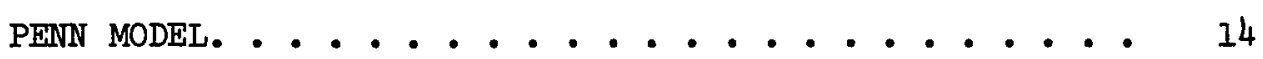

IV. KRAMERS-KRONIG RELATION AND THE $f$-SUM RULE . . . . 22

A. Verification and Application of the Kramers-Kronig

Relation................... 22

B. $f$-Sum Rule ............. 25

V. COMPARISON OF THEORETICAL CALCULATIONS WITH EXPERIMENTAL RESULTS . . . . . . . . . . . . . 27

VI. CONCLUDING REMARKS. . . . . . . . . . . 30

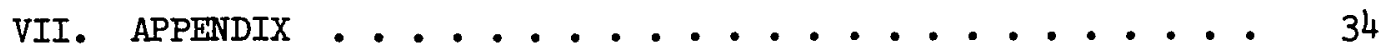

A. Calculation of $\epsilon_{2}(\omega)$ with $q$ Retained to Second

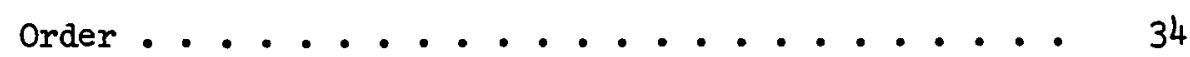

B. Three-Band, Penn-Type Model . . . . . . . . 37

VIII. ACKNOWLEDGEMENTS. ..................... 44

IX. REFERENCES. . . . . . . . . . . . . . 4 45

X. IIST OF TABLES. . . . . . . . . . . . . 47

XI. LIST OF FIGURES . . . . . . . . . . . . 50 
ABSTRACT

Improved expressions are obtained for the real, static part $\epsilon_{1}(0)$ and the imaginary part $\epsilon_{2}(w)$ of the dielectric constant of semiconductors in the long wavelength limit using the isotropic, nearlyfree-electron band approximation (Penn model). Earlier calculations of these functions do not satisfy the Kramers-Kronig relations and yield an excessively large result for the $f$-sum rule. The corrected expressions eliminate these inconsistencies. Values of the energy gap between the bonding and anti-bonding states are obtained for diamond, silicon and germanium. $\epsilon_{1}(\omega)$ is obtained from $\epsilon_{2}(\omega)$ through the use of the Kramers-Kronig relation. The theoretical curves for $\epsilon_{1}(\omega)$ and $\epsilon_{2}(\omega)$ are compared with experimental results. Corrected parameters for the Phillips-Van Vechten theory of ionicity are obtained through the use of the improved expression for $\epsilon_{1}(0)$. 


\section{INTRODUCTION}

Although detailed band structure calculations have been used to evaluate the dielectric constant for semiconductors with excellent results, ${ }^{1}$ the appeal of approximate calculations based on the isotropic, nearly-free-electron approximation remains strong due to physical insight gained from the simplicity of the analytical results. Prior to Penn's introduction of a nearly-free-electron model, isotropically extended to three dimensions ${ }^{2}$ (Penn model), only two crude approximations to the static $(\omega=0)$, real part of the dielectric constant $\epsilon_{1}(0, q)$ existed. The first approximation was to use the free electron gas value for $\epsilon_{1}(0, q) .3,4$ In the long wavelength $(q \rightarrow 0)$ limit, $\epsilon_{1}(0,0) \rightarrow \infty$ in the free electron gas model. However, the trend of the $q$ dependence of $\epsilon_{1}(0, q)$ is reasonably approximated away from $q=0$ by the free electron expression for materials whose $\epsilon_{1}(0,0)$ values are large. Then, Callaway ${ }^{5}$ refined the free electron gas approximation by inserting artificially an energy gap between the valence and conduction bands without, however, modifying the wave functions. Unlike these two approximations, the Penn model allows for the formation of standing waves at the zone edge and accounts for the contributions from Umklapp processes. Penn calculated $\epsilon_{1}(0, q)$ by approximating the matrix elements for the normal and Umklapp processes and obtained an expression for $\epsilon_{1}(0,0)$. He also demonstrated that Umklapp processes make the dominant contribution to $\epsilon_{1}(0, q 2)$ for $\operatorname{small} q$. 
Through the use of the Penn model, Srinivasan ${ }^{6}$ has performed calculations of $\epsilon_{1}(0, q)$ for even larger values of $q$ than were obtained by Penn. Bardasis and Hone ${ }^{7}$ have used the Penn model to calculate the imaginary part of the dielectric constant in the long wavelength $(q \rightarrow 0)$ limit, $\epsilon_{2}(\omega, 0)$. It can be shown that Penn's expression for $\epsilon_{1}(0,0)$ and Bardasis and Hone's expression for $\epsilon_{2}(m, 0)$ do not satisfy the Kramers-Kronig relation. 8

Phillips' theory of ionicity ${ }^{9}$ of the chemical bond in crystals is based upon the Penn model for semiconductors. In Phillips' theory the average energy gap for a semiconductor between the bonding and antibonding levels, $E_{g}$, is calculated from Penn's expression for $\epsilon_{1}(0,0)$. Empirical corrections for d-state effects have been determined by Van Vechten ${ }^{10}$ to allow the theory of ionicity to be extended to crystals where these effects play a role.

The failure of Penn's expression for $\epsilon_{1}(0,0)$ and Bardasis and Hone's expression for $\epsilon_{2}(w, 0)$ to satisfy the Kramers-Kronig relation, coupled with the fact that Phillips' theory is based upon the Penn result, provided the original motivation for the research upon which this thesis is based. The purpose of this thesis is to display improved expressions for the real and imaginary parts of the dielectric constant of semiconductors in the long wavelength $(q \rightarrow 0)$ limit through the use of the Penn model. Within the context of the Penn model no further approximations are used for the electron energy, and the matrix elements are more general than those employed by Penn. In chapter II the complex dielectric function for a crystal is expressed in the extended zone scheme by use of the self-consistent-field approach 
of Ehrenreich and Cohen. 11 In chapter III the expressions for $\epsilon_{1}(0,0)$ and $\epsilon_{2}(\omega, 0)$ are calculated. In chapter IV the Kramers-Kronig relation is shown to be satisfied by the improved expressions for $E_{1}(0,0)$ and $\epsilon_{2}(\omega, 0)$, which is the first time that this has been demonstrated for dielectric constants calculated by use of the Penn model. Then $\epsilon_{1}(\omega, 0)$ is calculated from $\epsilon_{2}(\omega, 0)$ by use of the Kramers-Kronig relation. Finally, it is shown that $E_{2}(\omega, 0)$ closely satisfies the $f$-sum rule. 12 In chapter $v$, values for the energy gap of diamond, silicon and germanium are obtained from experimental values of $\epsilon_{1}(0,0)$. Next, the theoretical calculations of $\epsilon_{1}(\omega, 0)$ and $\epsilon_{2}(\omega, 0)$ are compared with experimental results. In chapter VI the implications of the improved expression for $\epsilon_{1}(0,0)$ on Phillips' theory of ionicity are discussed. 
II. THE SELF-CONSISTENT-FIEID METHOD FOR

$$
\text { CALCULATION OF } E(\omega, q)
$$

To begin, the Ehrenreich and Cohen ${ }^{11}$ self-consistent-field approach will be used to calculate the general expression for $\epsilon(\omega, q)$ in the extended zone scheme. The single particle quantum mechanical analog to the Liouville equation is

$$
i \hbar \frac{\partial p}{\partial t}=[H, P] \text {, }
$$

which describes the response of any particle of the system to the: selfconsistent potential $V(r, t)$. $P$ is the single particle density operator. $H$ is the total one particle Hamiltonian

$$
H=H_{0}+V(x, t),
$$

where $H_{0}$ is the Hamiltonian for the electron in the unperturbed periodic lattice. $H_{0}|k\rangle=E_{k}|k\rangle$ where, in general, $|k\rangle$ is a Bloch function $|k\rangle=\Omega^{-\frac{1}{2}} e^{i k \cdot x} u_{k}(r)$. The density operator is expanded as $p=p^{(0)}+p^{(1)}$.

$$
\rho^{(0)}|k\rangle=f_{0}\left(E_{k}\right)|k\rangle,
$$

where $f_{0}\left(E_{k}\right)$ is the unperturbed distribution function. $V(r, t)$ can be Fourier analyzed in the form 


$$
V(\underline{r}, t)=\sum_{q^{\prime}} V\left(q_{\sim}^{\prime}, t\right) e^{-i \underline{q}^{\prime} \cdot \underline{r}}
$$

Equation (1) is linearized by neglecting products of the type $V p^{(1)}$. Ehrenreich and Cohen point out that this approximation is equivalent to first-order self-consistent perturbation theory. If one takes the matrix elements of $p^{(1)}$ between states $k_{1}$ and $k_{2}$, the following is obtained

$$
\begin{aligned}
& i \hbar \frac{\partial}{\partial t}\left\langle k_{1}\left|\rho^{(1)}\right| k_{2}\right\rangle=\left\langle k_{1}\left|\left[H_{0}, \rho^{(1)}\right]\right| k_{2}\right\rangle+\left\langle k_{1}\left|\left[v\left(r_{E}, t\right), \rho^{(0)}\right]\right| k_{2}\right\rangle \\
& =\left(E_{k_{1}}-E_{k_{2}}\right)\left\langle k_{k_{1}}\left|\rho^{(1)}\right| k_{2}\right\rangle+\left[f_{0}\left(E_{k_{2}}\right)-f_{0}\left(E_{k_{1}}\right)\right]\left\langle k_{1}\left|v\left(r_{2}, t\right)\right| k_{2}\right\rangle,
\end{aligned}
$$

where $r_{s}$ is the position operator.

$$
\left\langle k_{1}\left|v\left(\underline{r}_{0}, t\right)\right| \underline{k}_{2}\right\rangle=\sum_{q^{\prime}} v\left(q^{\prime}, t\right)\left\langle\underline{\sim}_{1}\left|e^{-i q^{\prime} \cdot r_{e}}\right| k_{2}\right\rangle .
$$

The potential $V$ consists of an external potential $V_{0}$ plus the screening potential $V_{S} \cdot V_{S}$ is related to the induced change in electron density,

$$
\begin{aligned}
\delta_{n} & =\operatorname{Tr}\left\{\delta\left(r_{e}-r\right) p^{(1)}\right\} \\
& =\sum_{\underline{k}_{2}}\left\langle\underline{k}_{2}\left|\frac{1}{I} \sum_{q^{\prime}} e^{i q^{\prime} \cdot\left(r_{e}-r\right)} p^{(1)}\right| k_{2}\right\rangle \\
& =\frac{1}{\Omega} \sum_{k_{1}, k_{2}, q^{\prime}} e^{-i q^{\prime} \cdot r}\left\langle\underline{k}_{2}\left|e^{i q^{\prime} \cdot r_{2}}\right| k_{1}\right\rangle\left\langle k_{1}\left|\rho^{(1)}\right| k_{2}\right\rangle,
\end{aligned}
$$


by Poisson's equation

$$
\nabla^{2} V_{s}=-4 \pi e^{2} \delta n
$$

where the electronic charge is-e. From equations (4), (7) and (8), the following expression is obtained,

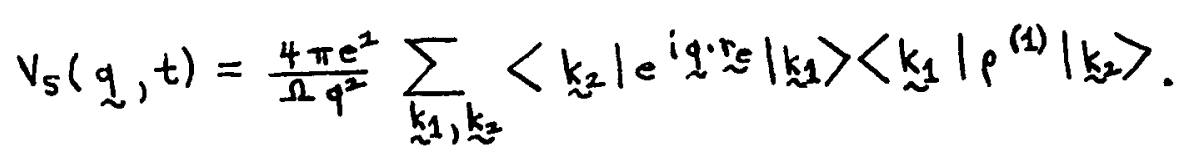

Imagine that the external potential $V_{0}(q, t)$ acts on the system with time dependence $e^{\alpha t} e^{i \omega t}$, where $\alpha \rightarrow 0$ corresponds to an adiabatic turning on of the perturbation. This potential polarizes the system. It follows from the definition of the dielectric constant, 13 that is, $\underset{\sim}{D}(\omega, q)=\in(\omega, q) \underset{\sim}{\mathcal{E}}(\omega, q)$, and from the Fourier analysis prescribed by equation (4) that

$$
\underset{\sim}{P}(\underline{q}, t)=\frac{1}{4 \pi}[\in(w, q)-1] \underset{\sim}{E}(\underline{q}, t) .
$$

The polarization $P(q, t)$ is related to the induced change in the electron density by $\nabla \cdot \underline{p}=e \delta n$ or $-i q \underline{\sim} \cdot \underset{\sim}{p}(\underline{q}, t)=e \delta n$. Since a longitudinal disturbance is being considered,

$$
-i q P(q, t)=e d n(q, t),
$$

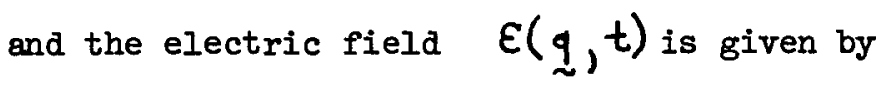


7

$$
\text { e } \varepsilon(\underline{q}, t)=-i q v(q, t)
$$

It is assumed that $\left\langle\underset{k_{1}}{ }\left|\rho^{(1)}\right| \underline{k}_{2}\right\rangle$ and $V_{s}(\underset{\sim}{q}, t)$ have the same time dependence as $V_{0}(q, t)$. If this assumption is used in equation (5), the following is obtained

$$
\left\langle{\underset{\sim}{k}}_{1}\left|p^{(1)}\right| k_{\alpha_{2}}\right\rangle=\lim _{\alpha \rightarrow 0} \frac{\left[f_{0}\left(E_{k_{2}}\right)-f_{0}\left(E_{k_{1}}\right)\right]}{E_{k_{2}}-E_{k_{1}}-\hbar \omega+i k_{\alpha}}\left\langle k_{1}\left|V\left(r_{e}, t\right)\right| k_{2}\right\rangle .
$$

From equations (7) and (13), the following expression is obtained

$$
\begin{aligned}
& \delta_{n}=\lim _{\alpha \rightarrow 0} \frac{1}{\Omega} \sum_{\underline{k}_{1}, \underline{k}_{2}, q^{\prime}} e^{-i q^{\prime} \cdot n} \frac{\left[f_{0}\left(E_{k_{2}}\right)-f_{0}\left(E_{k_{1}}\right)\right]}{E_{k_{2}}-E_{k_{1}}-\hbar \omega+i \hbar \alpha} \\
& x\left\langle k_{2}\left|e^{i q^{\prime} \cdot r_{s}}\right| k_{1}\right\rangle\left\langle\underset{k_{1}}{k_{1}}\left|v\left(r_{e}, t\right)\right| \underline{z}_{2}\right\rangle .
\end{aligned}
$$

Examine $\left\langle\underline{k_{2}}\left|e^{i q^{\prime} \cdot r_{e}}\right| \underline{k}_{1}\right\rangle$.

$$
\left\langle\underline{k_{2}}\left|e^{i q^{\prime} \cdot r_{e}}\right| \underline{k}_{1}\right\rangle=\frac{1}{\Omega} \int_{\Omega} d^{3} r_{e} e^{i\left(k_{1}+q^{\prime}-k_{2}\right) \cdot \underline{r}_{e}} u_{\underline{k}_{2}}^{*}\left(r_{e}\right) u_{k_{1}}\left(r_{e}\right) .
$$


The integral over the volume may be written as a sum of integrals over each unit cell. 14

$$
\left\langle k_{2}\left|e^{i q^{\prime} \cdot r_{e}}\right| k_{1}\right\rangle=\frac{1}{\Omega} \sum_{i} \int_{\substack{i t h \\ \text { unit } \\ \text { cell }}} d^{3} r_{e} e^{i\left(k_{1}+q^{\prime}-k_{2}\right) \cdot r_{e}} u_{k_{2}}^{*}\left(r_{e}\right) u_{k_{1}}\left(r_{2}\right)
$$

The functions $u_{k_{2}}^{*}\left(r_{e}\right)$ and $u_{k_{1}}\left(r_{e}\right)$ are periodic in the lattice, so the integral over the ith unit cell can be translated to the integral over the zeroth cell by changing variables to $\left(r_{e}-R_{i}\right)$. Then it can be shown that

$$
\begin{aligned}
& \left\langle\underline{k_{2}}\left|e^{i q^{\prime} \cdot r_{e}}\right| \underline{k}_{1}\right\rangle=\frac{N}{\Omega} \sum_{\underline{G}^{\prime}} \delta_{\underline{k}_{2}, \underline{k}_{1}+q^{\prime}+\underline{G}^{\prime}} \\
& x \int_{\substack{\text { zeroth } \\
\text { unit } \\
\text { cell }}} d^{3} r_{e} e^{i\left(k_{1}+q^{\prime}-k_{2}\right) \cdot r_{e}} u_{k_{2}}^{*}\left(r_{e}\right) u_{k_{1}}\left(r_{e}\right)
\end{aligned}
$$

because the integral is independent of the choice of unit cell. $N$ is the number of unit cells in the volume $\Omega . \quad G^{\prime}$ is one of the set of reciprocal lattice vectors required to satisfy the delta function. The matrix element can be written as follows:

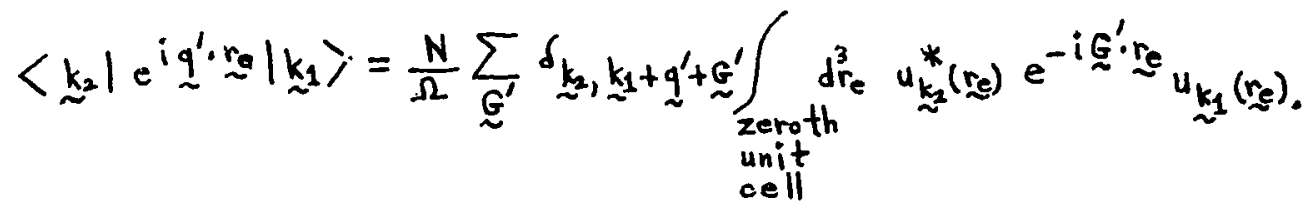

Since $u_{k_{2}}^{*}\left(r_{e}\right) e^{-i g^{\prime} \cdot r_{s}} u_{k_{1}}\left(r_{s}\right)$ is periodic in the lattice, the integral over the zeroth cell is equivalent to the integral over any unit cell 
9

and the matrix element becomes

$$
\begin{aligned}
& \left\langle\underline{k_{2}}\left|e^{i q^{\prime} \cdot r_{e}}\right|{\underline{k_{1}}}_{2}\right\rangle=\frac{1}{\Omega} \sum_{G^{\prime}} \delta_{\underline{k}_{2}, k_{1}+q_{1}^{\prime}+G^{\prime}} \int_{\Omega} d^{3} r_{e} u_{k_{2}}^{*}\left(r_{0}\right) e^{-i G^{\prime} \cdot r_{g}} u_{k_{1}}\left(r_{g}\right) \\
& =\sum_{\underline{G}^{\prime}} \delta_{\underline{k}_{2}, k_{1}+q^{\prime}+\underline{G}^{\prime}}\left\langle{\underline{k_{2}}}_{2}\left|e^{i q^{\prime} \cdot r_{2}}\right| \underline{\sim}_{1}\right\rangle .
\end{aligned}
$$

(17)

From equations (14), (4) and (17), one finds

$$
\begin{aligned}
& \delta_{n}=\lim _{\alpha \rightarrow 0} \frac{1}{\Omega} \sum_{\sum_{\alpha_{1}, k_{2}}} \sum_{q^{\prime}, q_{2} G_{\sim}^{\prime}, G} \delta_{k_{2}, k_{1}+q^{\prime}+G^{\prime}}\left\langle k_{2}\left|e^{i q^{\prime} \cdot r_{e}}\right| k_{k_{1}}\right\rangle \\
& X \frac{e^{-i q^{\prime} \cdot r}\left[f_{0}\left(E_{k_{2}}\right)-f_{0}\left(E_{k_{1}}\right)\right]}{E_{k_{2}}-E_{k_{1}}-\hbar \omega+i \hbar \alpha} \quad \delta_{k_{2}, k_{1}}+q+G \\
& x\left\langle\underset{k_{1}}{k_{1}}\left|e^{-i \underline{q} \cdot r_{e}}\right| \underline{k}_{2}\right\rangle v(\underline{q}, t)
\end{aligned}
$$

which reduces to the expression

$$
\begin{aligned}
& \delta_{n}=\lim _{\alpha \rightarrow 0} \frac{1}{\Omega} \sum_{k_{1_{1}}, k_{2}} \sum_{\underline{q}^{\prime}, G^{\prime}, G} \frac{e^{-i q^{\prime} \cdot r}\left[f_{0}\left(E_{k_{2}}\right)-f_{0}\left(E_{k_{2}}\right)\right]}{E_{k_{2}}-E_{k_{1}}-\hbar w+i \hbar \alpha}
\end{aligned}
$$

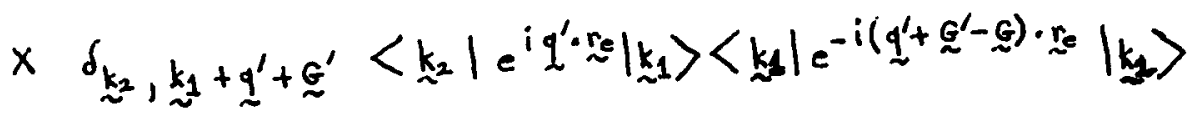

$$
\begin{aligned}
& x \quad V\left(q^{\prime}+\underline{\sigma}^{\prime}-\underset{\sim}{G}, t\right) .
\end{aligned}
$$


Through the use of equations (4) and (8), it can also be shown that

$$
v_{s}(q, t)=\frac{4 \pi e^{2}}{q^{2}} \operatorname{sn}(q, t)
$$

Large values of $q$ in $v_{s}(q, t)$ or $\delta_{n}(q, t)$ are not of interest because they would contribute spacially rapid fluctuations in $V_{s}\left(r_{e}, t\right)$ and $\delta_{n}\left(r_{e}, t\right)$ that average out in the sum over $q$. Likewise, large values of $q$ in $V\left(r_{f}, t\right)=\sum_{q} V(q, t) e^{-i q \cdot r_{j}}$ are not of interest. $\quad V\left(q^{\prime}+G^{\prime}-G, t\right)$ in equation (19) for $G^{\prime} \neq \underline{G}$ would be associated with a component that is spatially a rapidly varying function and also tends to be averaged out over spatial regions of interest. This is equivalent to neglecting local field corrections as Fhrenreich and Cohen ${ }^{11}$ have done. Therefore, it is required that $G=G^{\prime}$ and $\delta n$ becomes

$$
\begin{aligned}
& \delta_{n}=\lim _{\alpha \rightarrow 0} \frac{1}{\Omega} \sum_{\underline{k}_{1}, k_{2}, q_{2}^{\prime}, G^{\prime}} \frac{e^{-i q^{\prime} \cdot r}\left[f_{0}\left(E_{k_{2}}\right)-f_{0}\left(E_{k_{1}}\right)\right]}{E_{k_{2}}-E_{k_{1}}-\hbar \omega+i \hbar \alpha} V\left(q_{1}^{\prime}, t\right)
\end{aligned}
$$

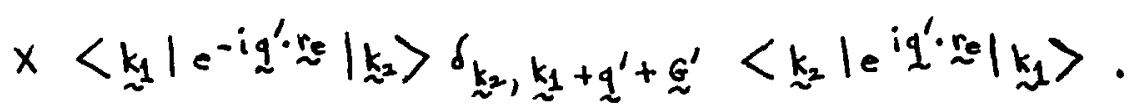

In can be written as

$$
\begin{aligned}
& d n=\lim _{\alpha \rightarrow 0} \frac{1}{\Omega} \sum_{\left.\underline{k_{1}} \underline{1}^{\prime}\right)^{\prime}} \frac{e^{-i q^{\prime} \cdot r}\left[f_{0}\left(E_{k_{1}+q^{\prime}+G^{\prime}}\right)-f_{0}\left(E_{k_{1}}\right)\right]}{E_{k_{1}+q^{\prime}}+\underline{G}^{\prime}-E_{k_{1}}-\hbar_{w}+i \hbar \alpha} V\left(q_{\alpha}^{\prime}, t\right) \\
& x\left|\left\langle k_{1}\left|e^{-i q^{\prime} \cdot r_{e}}\right| k_{q}+q^{\prime}+G^{\prime}\right\rangle\right|^{2} \text {. }
\end{aligned}
$$


For the ease of writing, $k_{1}$ can be replaced by $k, r_{c}$ by $\mathfrak{r}$ and $\mathfrak{G}^{\prime}$ by $G$.

$$
\begin{aligned}
& \text { Then } \delta_{n}(q, t) \text { is } \\
& \delta_{n}(q, t)=\lim _{\alpha \rightarrow 0} \frac{V(q, t)}{\Omega} \sum_{\underline{k}, \underline{G}} \frac{\left[f_{1}\left(E_{\underline{\underline{\alpha}}+q+g}\right)-f_{0}\left(E_{k}\right)\right]}{E_{\underline{k}+q}+\underline{\sigma}-E_{k}-\hbar \omega+i \hbar \alpha} \\
& x\left|\left\langle k\left|e^{-i q \cdot r}\right| \underline{k}+q+g\right\rangle\right|^{2} \text {. }
\end{aligned}
$$

From equations (10), (11), and (12), the following expression for $\epsilon(\omega, \underset{\sim}{q})$ is obtained,

$$
\epsilon(\omega, q)=1-\frac{4 \pi e^{2} \delta n(q, t)}{q^{2} V(\underset{q}{q}, t)} .
$$

The combination of equations (23) and (24) yields

$$
\begin{aligned}
& \epsilon(\omega, q)=1-\lim _{\alpha \rightarrow 0} \frac{4 \pi c^{2}}{q^{2} \Omega} \sum_{\underline{k}, \xi} \frac{\left[f_{0}\left(E_{k+q}+\underline{q}\right)-f_{0}\left(E_{k}\right)\right]}{E_{k}+q+G-E_{k}-\hbar \omega+i \hbar \alpha} \\
& x \mid\langle k| e^{-\left.i q \cdot a|k+q+G\rangle\right|^{2},}
\end{aligned}
$$

in the extended zone. ${ }^{15}$

The complex dielectric function is defined here as

$$
\epsilon(w, q)=\epsilon_{1}(w, q)-i \epsilon_{2}(\omega, q),
$$


so that $E_{2}(w, q)$ will be a positive quantity. Equations (25) and (26) give final expressions for $\epsilon_{1}(\omega, q)$ and $\epsilon_{2}(\omega, q)$,

$$
\begin{aligned}
& E_{1}(\omega, q)=1-\frac{4 \pi e^{2}}{q^{2} \Omega} \sum_{\underline{k}, \underline{G}} \mid\langle k| e^{-\left.i q \cdot I|\underline{k}+q+G\rangle\right|^{2}} \\
& x\left[f_{0}\left(E_{\underline{k}+q}+G\right)-f_{0}\left(E_{\underline{k}}\right)\right] P\left(\frac{1}{E_{k}+q+G-E_{k}-\hbar \omega}\right),
\end{aligned}
$$

where $P($ ) indicates the principal value, and

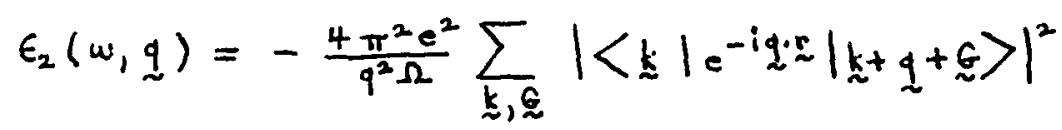

$$
\begin{aligned}
& x\left[f_{0}\left(E_{\underline{k}+q+G}\right)-f_{0}\left(E_{\underline{q}}\right)\right] \delta\left(E_{\underline{k}+\underline{q}+\underline{G}}-E_{\underline{\underline{k}}}-\hbar \omega\right),
\end{aligned}
$$

where $\delta()$ is the Dirac delta function. These expressions for $\epsilon_{1}(\omega, q)$ and $\epsilon_{2}(w, q)$ have been derived starting from a one electron Hamiltonian so some electron correlation effects are neglected. Also, local field corrections have been neglected. Both of these approximations restrict the validity of $\epsilon_{1}(\omega, q)$ and $\epsilon_{2}(\omega, q)$ to $q<\frac{2 \pi}{a}$ where $q$ is a lattice parameter. The neglect of electron correlations also places a restriction on the frequency where $\epsilon_{1}(\omega, q)$ and $\epsilon_{2}(\omega, q)$ can be considered good approximations. It is thought that at frequencies on the 
order of the plasma frequency the expressions for $\epsilon_{1}(\omega, q)$ and $\epsilon_{2}(\omega, \underset{\sim}{q})$ may be subject to inaccuracies. 


\section{CALCULATION OF $\epsilon_{1}(0)$ AND $\epsilon_{2}(\omega)$ \\ USING THE PENN MODEL}

At zero temperature, $f_{0}\left(E_{t}\right)$ becomes the occupation number $N_{k}$. Equations (27) and (28) then become

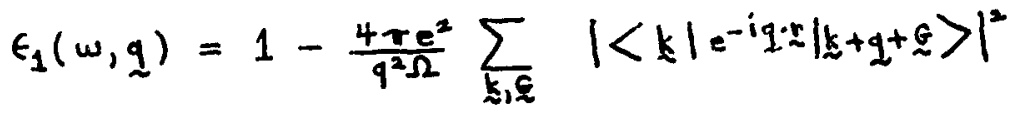

$$
\begin{aligned}
& x\left[N_{\underline{k}+q}+\underline{\sigma}-N_{\underline{k}}\right] P\left(\frac{1}{E_{\underline{k}+q}+\underline{q}-E_{\underline{z}}-\hbar_{\omega}}\right)
\end{aligned}
$$

and

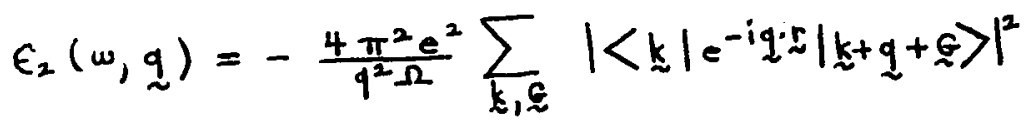

$$
\begin{aligned}
& x\left[N_{\underline{k}+q}+\underline{q}-N_{\underline{k}}\right] \delta\left(E_{\underline{\underline{k}}+q+q}-E_{\underline{\underline{z}}}-\hbar \omega\right),
\end{aligned}
$$

respectively.

The quantities of interest are $\epsilon_{1}(0, q)$ and $\epsilon_{2}(w, q)$ in the limit as $q$ goes to zero. The Penn model ${ }^{2}$ can be used to obtain closed form expressions for these quantities from equations (29) and (30). The model is equivalent to the nearly-free-electron model (see figure 1), isotropically extended to three dimensions. The energies of the two 
bands are given by

$$
E_{\underline{k}}^{ \pm}=\frac{1}{2}\left\{E_{\underline{k}}^{0}+E_{\underline{X}^{\prime}}^{0} \pm\left[\left(E_{\underline{k}}^{0}-E_{\underline{k}^{\prime}}^{0}\right)^{2}+E_{g}^{2}\right]^{\frac{1}{2}}\right\},
$$

where $E_{k}^{0}=\frac{k^{2} k^{2}}{2 m}$ and $\underline{k}^{\prime}=\underset{\sim}{k}-2 k_{F} \hat{k} \cdot k_{F} \hat{k}$ is the Fermi wave vector. The wave functions are

$$
\psi_{\underline{k}}^{ \pm}(x)=\frac{e^{i k \cdot x}}{\Omega^{1 / 2}}\left\{\frac{1-\beta_{t}^{ \pm} e^{-i 2 k_{F} \hat{k} \cdot r}}{\left[1+\left(\beta_{t}^{ \pm}\right)^{2}\right]^{1 / 2}}\right\}
$$

where $\beta_{\underline{k}}^{ \pm}=\frac{E_{g}}{E_{\underline{k}}^{\cdot}-E_{\underline{k}^{\prime}}^{ \pm} \pm\left[\left(E_{\underline{k}}^{\prime}-E_{L^{\prime}}^{\prime}\right)^{2}+E_{g}^{1}\right]^{\frac{1}{2}}}$. In the preceding expressions, the - and + superscripts refer to $\underset{\sim}{k}$ in the first Brillouin zone (Jones zone) and $k$ in the second Brillouin zone, respectively.

$$
\text { Since } \epsilon_{1}(0, q) \text { and } \epsilon_{2}(w, q) \text { are of interest in the limit as }
$$
$q$ goes to zero, for a given ${\underset{\sim}{1}}_{1}$ then $k_{2}=k_{1}+q$ does not have an independent reciprocal lattice vector if the angle between $\underline{k}_{1}$ and $k_{2}$ is smaller than the smallest angle between the reciprocal lattice vectors of interest in a real crystal. This is known as the Srinivasan condition. ${ }^{6}$ Srinivasan argues that in a real crystal there are only a discrete number of reciprocal lattice vectors, so assigning separate reciprocal lattice vectors to all $k_{1}$ and $k_{2}$ is not physical. In the following calculations, this condition should definitely hold since $q$ is always small and will finally be allowed to go to zero. There are two values of $G$ associated with the Penn model: $G=0$ which 
16

corresponds to the normal process, and $\underset{\sim}{G}=-2 k_{F} \hat{k}$ which corresponds to the Umklapp process. Since the Srinivasan condition is used, $k$ and $k+q$ have the same reciprocal lattice vector, $-2 k_{p} \hat{k}$ (see figuse 2).

If equations (31) and (32) are substituted in equation (29), the following expression is obtained for $\epsilon_{1}(0)$ :

$$
\begin{aligned}
& \epsilon_{1}(0)=1-\lim _{q \rightarrow 0} \frac{4 \pi e^{2}}{q^{2} \Omega} \sum_{\underline{k}}\left[N_{k+q}-n_{k}\right] \frac{\left|\left\langle k\left|e^{-i q \cdot i}\right| k+q\right\rangle\right|^{2}}{E_{k+q}^{+}-E_{k}^{-}} \\
& -\lim _{q \rightarrow 0} \frac{4 \pi e^{2}}{q^{2} \Omega} \sum_{\underline{k}}\left[N_{k^{\prime}+q}-N_{k}\right] \frac{\left|\left\langle k\left|e^{-i q \cdot z}\right| k^{\prime}+q\right\rangle\right|^{2}}{E_{\underline{k}^{\prime}+q}^{+}-E_{\underline{k}}^{-}} .
\end{aligned}
$$

For the second term, two possibilities exist that give rise to nonzero contributions to $\epsilon_{1}(0): \underline{k}$ in the first Brillouin zone and $\underline{k}+q$ in the second zone, and vice versa. A similar situation exists for the third term. Therefore, $\epsilon_{1}(0)$ can be written as

$$
\begin{aligned}
& \epsilon_{1}(0)=1+\lim _{q \rightarrow 0} \frac{4 \pi e^{2}}{q^{2} \Omega} \sum_{\underline{k}} H_{k}\left[1-H_{k+q}\right] \frac{\left|\left\langle k \mid e^{-i q \cdot k \mid k+q}\right\rangle\right|^{2}}{E_{k+q}^{+}-E_{k}^{-}} \\
& -\lim _{q \rightarrow 0} \frac{4 \pi e^{2}}{q^{2} \Omega} \sum_{k} N_{k+q}\left[1-N_{k}\right] \frac{\left|\left\langle\underline{k}\left|e^{-i q \cdot \Sigma}\right| \underline{k}+q\right\rangle\right|^{2}}{E_{k+q}^{t}-E_{k}} \\
& +\lim _{q \rightarrow 0} \frac{4 \pi e^{2}}{q^{2} \Omega} \sum_{k} N_{k}\left[1-N_{k^{\prime}+q}\right] \frac{\left|\left\langle\underline{k}\left|e^{-i q \cdot r}\right| \underline{k}^{\prime}+q\right\rangle\right|^{2}}{E_{\underline{k}+q}^{+}-E_{k}^{\prime}} \\
& -\lim _{q \rightarrow 0} \frac{4 \pi e^{2}}{q^{2} \Omega} \sum_{k} N_{k^{\prime}+q}\left[1-N_{k}\right] \frac{\left|\left\langle k\left|e^{-i q \cdot r}\right| k^{\prime}+q\right\rangle\right|^{2}}{E_{k^{\prime}+q}^{+}-E_{k}^{\prime}}
\end{aligned}
$$


17

In the second term of the above equation, let $\underset{\sim}{\underline{k}} \rightarrow-\underline{k}-q$; and in the fifth term, let $\underline{k} \rightarrow-\underline{k}-\underline{q}+2 k_{F} \hat{k}$, remembering that $\underline{k}^{\prime}=\underline{k}-2 k_{F} \hat{k}$. Since $E_{\underline{k}}$ is even in $k, E_{-k-q}=E_{\underline{k}+q}$ and $E_{-k^{\prime}-q}=E_{\underline{k}}+q$. Likewise, the same thing is true for $N_{\mathfrak{x}}$.

$$
\begin{aligned}
\left\langle-\underline{k}-q-G\left|e^{-i q \cdot r}\right|-\underline{k}\right\rangle & =\int_{\Omega} d^{3} r \psi_{-k-q-G}^{*}(r) e^{-i q \cdot r \psi_{-k}(x)} \\
& =\int_{\Omega} d^{3} r \psi_{-k-q-G}^{*}(-r) e^{i q \cdot r} \psi_{-k}(-r),
\end{aligned}
$$

where $\stackrel{r}{\sim}-\underline{r}$ in the integral. Since the Hamiltonian is invariant under space inversion, $\psi_{-k}(-r)=\psi_{k}(r)$, apart from a phase factor, 16

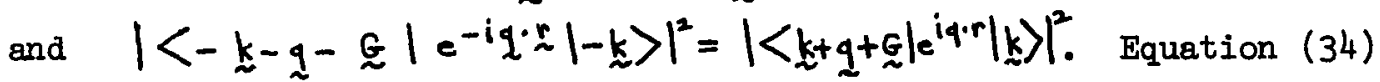
can now be written as

$$
\begin{aligned}
\epsilon_{1}(0) & =1+\lim _{q \rightarrow 0} \frac{8 \pi e^{2}}{q^{2} \Omega} \sum_{k} N_{k}\left[1-N_{k+q}\right] \frac{\left|\left\langle\underline{k}\left|e^{-i q \cdot t}\right| \underline{k}+q\right\rangle\right|^{2}}{E_{\underline{k}+q}^{+}-E_{\underline{k}}^{-}} \\
+ & \lim _{q \rightarrow 0} \frac{8 \pi e^{2}}{q^{2} \Omega} \sum_{\underline{k}} N_{k}\left[1-N_{k^{\prime}+q}\right] \frac{\left|\left\langle\underline{k}\left|e^{-i q \cdot q}\right| k^{\prime}+q\right\rangle\right|^{2}}{E_{\underline{k}^{\prime}+q}^{+}-E_{\underline{k}}^{-}} .
\end{aligned}
$$

Starting with equation (30), (31) and (32), by a similar procedure it can be shown that

$$
\begin{aligned}
& \epsilon_{2}(w)=\lim _{q \rightarrow 0} \frac{4 \pi^{2} e^{2}}{q^{2} \Omega}\left\{\sum_{k} N_{k} \cdot\left[1-N_{k+q}\right]\left|\left\langle k \mid e^{-i q \cdot r \mid k+q}\right\rangle\right|^{2}\right.
\end{aligned}
$$

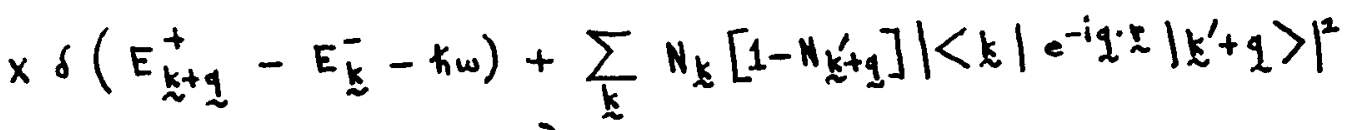

$$
\begin{aligned}
& \left.x \delta\left(E_{L^{\prime}+q}^{+}-E_{\underline{k}}^{-}-\hbar w\right)\right\} \text {, }
\end{aligned}
$$


18

where $\omega$ is restricted to positive frequencies. $N_{k}=1$ for $k$ in the first Brillouin zone and $N_{k}=0$ for $k$ elsewhere.

The nearly-free-electron model, isotropically extended to three dimensions, does not correspond to any real crystal structure. However, to perform the integrals for the calculation of the matrix elements, the properties of a real crystal structure are assumed.

Evaluation of the matrix elements yields

$$
\left|\left\langle\underline{k}\left|e^{-i q \cdot q}\right| \underline{k}+q\right\rangle\right|^{2}=\frac{\left[1+\beta_{k}^{-} \beta_{k+q}^{+}\right]^{2}}{\left[1+\left(\beta_{k}^{-}\right)^{2}\right]\left[1+\left(\beta_{k+q}^{+}\right)^{2}\right]}
$$

and

$$
\left|\left\langle\underline{k}\left|e^{-i q \cdot \underline{r}}\right| \underline{k}^{\prime}+q\right)\right|^{2}=\frac{\left[\beta_{\underline{k}}^{-}+\beta_{\underline{k}^{\prime}+q}^{+}\right]^{2}}{\left[1+\left(\beta_{\underline{k}}^{-}\right)^{2}\right]\left[1+\left(\beta_{\underline{L}+q}^{+}\right)^{2}\right]},
$$

where use has been made of $\int_{\Omega} d^{3} r e^{i \xi \cdot r}=0$ for $G \neq 0$. If the expressions in equations (35) and (36) are examined as $q$ tends to zero, the leading term goes as

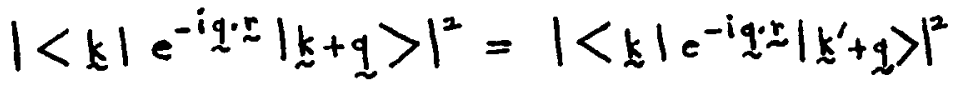

$$
\begin{aligned}
& \cong \frac{z^{2} \Delta^{2} \eta^{2}}{4\left[x^{2}+\Delta^{2}\right]^{2}} \text {, }
\end{aligned}
$$

where $z=\cos \theta, \theta$ is the angle between $\underset{\sim}{k}$ and $q, x=1-\frac{k}{k_{F}}$, $\Delta=\frac{E_{g}}{4 E_{F}}, \eta=\mathcal{K}_{K_{F}}$ and $E_{F}=\frac{\hbar^{2} k_{F}^{2}}{2 m}$. 
The normal process does not contribute to $\epsilon_{1}(0)^{6}$ or $\epsilon_{2}(w), 7$ so equations $(33)$ and $(34)$ become

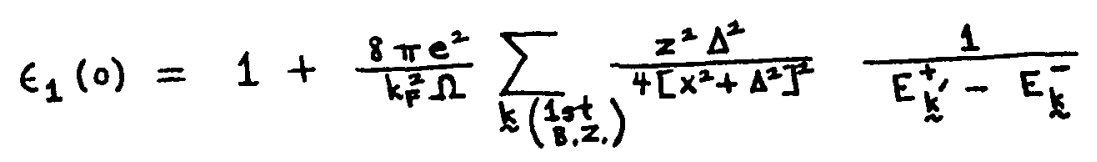

and

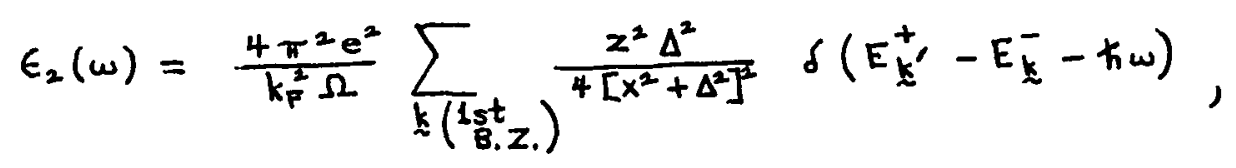

respectively, where the limit as $q \rightarrow 0$ has been taken. The sum on $\mathrm{k}$ over the first Brillouin zone is converted into an integral, and the integrals are performed exactly to give:

$$
\epsilon_{1}(0)=1+\frac{2}{3} \frac{E_{1}^{2}}{E_{j}^{2}}\left[\left(1+\Delta^{2}\right)^{\frac{1}{2}}-\Delta\right]
$$

and

$$
\epsilon_{2}(E)=\frac{\pi}{2} \frac{E_{p}^{2}}{E^{5}} \frac{\left[E_{g}-\Delta\left(E^{2}-E_{g}^{2}\right)^{1 / 2}\right]^{2}}{\left(E^{2}-E_{g}^{2}\right)^{1 / 2}}
$$


where $E_{p}=\left[\frac{4 \hbar^{2} \pi e^{2} n}{m}\right]^{1 / 2}$ is the plasma energy, and $E=\hbar \omega$. Equation (4I) holds for $E_{g} \leq E \leq 4 E_{F}\left(1+\Delta^{2}\right)^{1 / 2}$ and $\epsilon_{2}=0$ for other values of $E$. The procedure for setting $q=0$ directly in the delta function in equation (39) is somewhat facile, but it can be shown (see Appendix A) that if $q$ is retained to second order in the delta function, then an expression similar to equation (4I) is obtained that holds for the energy range $E_{g}<E \leq 4 E_{F} \cdot\left(1+\Delta^{2}\right)^{\frac{1}{2}}$.

Equation (4I) differs from the result given by Penn, ${ }^{2} \epsilon_{1}(0)=$ $1+\frac{E_{p}^{2}}{E_{g}^{2}}\left(1-\Delta+\frac{1}{3} \Delta^{2}\right)$. The factor of $\frac{2}{3}$ appearing in the second term of equation (40) is of particular importance in calculations of a homopolar energy gap, $E_{g}$, from measured values of $\epsilon_{1}(0)$. The difference arises because of the two approximations that Penn employs. The first approximation is that the electron energy is the free electron energy for $k<(1-\Delta) k_{F}$ and for $k>(1+\Delta) k_{F}$; but $E_{k}^{-} \approx(1-\Delta) E_{F}$ for $(1-\Delta) k_{F}<k<k_{F}$ and $E_{k}^{+} \approx(1+\Delta) E_{F}$ for $k_{F}<k<(1+\Delta) k_{F}$. Penn indicates that this approximation will introduce an error in $\epsilon_{1}(q)$ that is of order $\frac{E_{g}}{E_{F}}$ which is sizeable. The second approximation is that Penn uses the matrix element values at the Brillouin zone boundary to perform his calculations. These two approximations tend to maximize the value of $\epsilon_{1}(0)$.

Equation (4I) differs from the Bardasis and Hone result ${ }^{7}$ by a factor of $\frac{1}{2}$, but has the same functional dependence on $E_{p}, E_{g}, E_{F}$ and $E$. In the next chapter, additional evidence is presented to support the reduction by $\frac{1}{2}$ in the strength of the absorption. Furthermore, the expression for $\epsilon_{2}(\omega)$ given by Bardasis and Hone holds for the same region of frequency (or energy) as equation (4I), but the 
derivation by those authors does not make this constraint on the result explicit. 
IV. KRAMERS-KRONIG RELATION AND THE $f$-SUM RULE

A. Verification and Application of the Kramers-Kronig Relation

The Kramers-Kronig relation of interest can be shown to be

$$
\epsilon_{1}(E)-1=\frac{2}{\pi} P \int_{0}^{\infty} \frac{\epsilon_{2}\left(E^{\prime}\right) E^{\prime} d E^{\prime}}{E^{\prime 2}-E^{2}}
$$

and for $E=0$,

$$
\epsilon_{1}(0)-1=\frac{2}{\pi} P \int_{0}^{\infty} \frac{\epsilon_{2}\left(E^{\prime}\right) d E^{\prime}}{E^{\prime}} \text {. }
$$

Since the delta function in $\epsilon_{2}\left(E^{\prime}\right)$ restricts its non-zero range to $E_{g}<E^{\prime} \leq 4 E_{F}\left(1+\Delta^{2}\right)^{1 / 2}$, the principal value sign can be dropped. From equations (39) and (43),

$$
\begin{aligned}
& \epsilon_{1}(0)-1=\frac{2}{\pi} \frac{e^{2} \Delta^{2} k_{F}}{3} \int_{0}^{\infty} \int_{0}^{1} \frac{(1-x)^{2} d x}{\left[x^{2}+\Delta^{2}\right]^{2}} \frac{d E^{\prime}}{E^{\prime}} \\
& x \delta\left[4 E_{F}\left(x^{2}+\Delta^{2}\right)^{1 / 2}-E^{\prime}\right] .
\end{aligned}
$$

The following expression is then obtained for $\epsilon_{1}(0)$ : 


$$
\epsilon_{1}(0)=1+\frac{2}{3} \frac{E_{p}^{2}}{E_{g}^{2}}\left[\left(1+\Delta^{2}\right)^{1 / 2}-\Delta\right],
$$

which is in complete agreement with equation (40). Thus, the KramersKronig relation is satisfied by our expressions for $\epsilon_{1}(0)$ and $\epsilon_{2}(\omega)$, a condition that is not met by the expressions for the dielectric constants derived previously using the Penn model. It can be shown easily, for example, that Penn's expression for $\epsilon_{1}(0)$ and Bardasis and Hone's expression for $\epsilon_{2}$ (E) do not satisfy equation (43).

For $E<E_{g}\left(\omega<\omega_{g}\right)$, the Kramers-Kronig relation can be used to obtain $\epsilon_{1}(E)$. From equations (4I) and (42), it follows that

$$
\epsilon_{1}(E)-1=\frac{e^{2} \Delta^{2} k_{F}}{6 \pi E_{F}} \int_{0}^{1} \frac{(1-x)^{2} d x}{\left[x^{2}+\Delta^{2}\right]^{3 / 2}\left[x^{2}+\Delta^{2}-\left(\frac{E}{4 E_{F}}\right)^{2}\right]},
$$

where the principal value sign has been dropped because the integral exists. $E_{1}(E)$ can be written as

$$
\epsilon_{1}(E)=1+\frac{2}{3} \frac{E_{p}^{2}}{E_{j}^{2}} I(E),
$$


where

$$
I(E)=\frac{3}{2} \Delta^{2} \int_{0}^{1} \frac{(1-x)^{2} d x}{\left[x^{2}+\Delta^{2}\right]^{3 / 2}\left[\left(\frac{x}{\Delta}\right)^{2}+1-\left(\frac{E}{E_{y}}\right)^{2}\right]}
$$

This expression has been evaluated numerically for selected values of

$\Delta$ (see chapter $\mathrm{V}$ ).

For $E_{g}<E<4 E_{F}\left(1+\Delta^{2}\right)^{\frac{1}{2}}$, equation (46) can be written as

$$
E_{1}(E)-1=\frac{e^{2} \Delta^{2} k_{F}}{6 \pi E_{F}} P \int_{0}^{1} \frac{(1-x)^{2} d x}{\left[x^{2}+\Delta^{2}\right]^{3 / 2}\left[x^{2}-a^{2}\right]},
$$

where $\quad d=\left[\left(\frac{E}{4 E_{F}}\right)^{2}-\Delta^{2}\right]^{\frac{1}{2}} \quad$ and $\quad 0<d<1 \quad$. After a partial fraction expansion, the integration can be performed; that is, the principal value exists. Finally, $\epsilon_{1}(E)$ can be shown to be

$$
\begin{aligned}
& E_{1}(E)=1+\frac{E_{y}^{2}}{E_{g}^{2}}\left\{\frac{\Delta^{2}}{\left(1+\Delta^{2}\right)^{1 / 2}}-\frac{\Delta^{2}\left(2 \Delta^{2}-2 \Delta\left(1+\Delta^{2}\right)^{4 / 2}+q^{2}+1\right)}{\left(a^{2}+\Delta^{2}\right)\left(1+\Delta^{2}\right)^{1 / 2}}+\right. \\
& \frac{\Delta^{*}}{\left(a^{2}+\Delta^{2}\right)^{3 / 2}}\left[\log \left(\frac{a^{2}\left(\left(1+\Delta^{2}\right)^{1 / 2}\left(a^{2}+\Delta^{2}\right)^{\frac{1}{2}}+\Delta^{2}-a\right)\left(\left(1+\Delta^{2}\right)^{1 / 2}\left(a^{2}+\Delta^{2}\right)^{\frac{1}{2}}+\Delta^{2}+q\right)}{(1-q)(1+d)\left(\Delta\left(a^{2}+\Delta^{2}\right)^{1 / 2}+\Delta^{2}\right)^{2}}\right)\right. \\
& \left.\left.-\frac{1}{2}\left(a+\frac{1}{a}\right) \log \left(\frac{(1+a)\left(\left(1+\Delta^{2}\right)^{\frac{1}{2}}\left(a^{2}+\Delta^{2}\right)^{\frac{1}{2}}+\Delta^{2}+a\right)}{(1-a)\left(\left(1+\Delta^{2}\right)^{1 / 2}\left(a^{2}+\Delta^{2}\right)^{\frac{1}{2}}+\Delta^{2}-a\right)}\right)\right]\right\} .
\end{aligned}
$$


Equation (50) can be evaluated for a given $E_{g}$ (or $\Delta$ ) and various values of $E$. However, extensive calculations were not performed for values of $E$ outside the range in which equation (50) holds since experimental results for the semiconductors of interest do not exist for an energy (or frequency) this large. In addition, a sufficient range of energy has been covered to establish any trends for $\epsilon_{1}(E)$. B. $f$-Sum Rule

The $f$-sum rule can be written in the form

$$
\frac{2}{\pi} \int_{0}^{\infty} E \epsilon_{2}(E) d E=E_{p}^{2}
$$

The expression for $\epsilon_{2}(w)$ obtained earlier (equation 4I) can be used to sum the oscillator strength and obtain

$$
\begin{aligned}
& \frac{2}{\pi} \int_{0}^{\infty} E \epsilon_{2}(E) d E=E_{p}^{2}\left\{\left(1+\Delta^{2}\right)^{\frac{1}{2}}-2 \Delta+\right. \\
& \left.\Delta^{2} \log \left[\frac{1+\left(1+\Delta^{2}\right)^{1 / 2}}{\Delta}\right]\right\}
\end{aligned}
$$

For typical semiconductors, $\Delta$ is usually near 0.1. Therefore, this value is used to obtain $\frac{2}{\pi} \int_{0}^{\infty} E \epsilon_{2}(E) d E=0.835 E_{p}^{2}$. Evidently the simple two band model proposed by Penn artifically reduces the total oscillator strength by eliminating the possibility of transitions to other bands. To estimate the importance of additional bands, a nearly-free-electron, three-band model that is isotropically extended to three dimensions has been used to determine the effect of including 
more energy states on the $f$-sum rule (see Appendix B). For $\Delta=0.1$, essentially the same result is obtained which implies that the inclusion of more energy states does not improve the situation. A plausible explanation for why the $f$-sum rule is not satisfied exactly is that the nearly-free-electron model yields approximate wave functions and energies because a full set of reciprocal lattice vectors has not been employed.

The Bardasis and Hone result for $\epsilon_{2}(\omega)$ is a factor of two larger than ours, and for $\Delta=0.1$ gives $\frac{2}{\pi} \int_{0}^{\infty} E \epsilon_{2}(E) d E=1.67 E_{p}^{2}$, a result that is in significant disagreement with the $f$-sum mule. If the range of energy over which the expression for $\epsilon_{2}(E)$ is valid is not restricted when conducting the integral, the discrepancy is even larger. The present calculation brings $\epsilon_{2}(E)$ in closer agreement with the $f$-sum rule but the total oscillator strength is too small in the Penn model for the $f$-sum rule to be satisfied exactly. 
V. COMPARISON OF THEORETICAL CALCULATIONS

WITH EXPERIMENTAL RESULTS

To obtain $E_{g}$, the average energy gap between the bonding and the antibonding levels, equation (40) can be approximated as

$$
\epsilon_{1}(0) \cong 1+\frac{2}{3} \frac{E_{p}^{2}}{E_{g}^{2}}\left[1-\left(\frac{E_{g}}{4 F_{f}}\right)\right] \text {. }
$$

Experimental values of $\epsilon_{1}(0)$ are 5.7, 12 and 16 for diamond, silicon and germanium, respectively. From equation (53), $E_{g}$ is calculated to be $11.2 \mathrm{eV}$ for diamond and $3.92 \mathrm{eV}$ for silicon. If Van Vechten's empirical value ${ }^{10} \mathrm{D}$ is included to take into account d-state effects, then $\epsilon_{1}(0)$ becomes

$$
\epsilon_{1}(0) \cong 1+\frac{2}{3} \frac{D E_{p}^{2}}{E_{g}^{2}}\left[1-\left(\frac{E_{g}}{4 E_{F}}\right)\right] .
$$

For diamond and silicon, $D=1.0$. However, for germanium $D=1.25$ which results in a value of $3.53 \mathrm{eV}$ for $E_{g}$. These values for $E_{g}$ are lower than the values of $13.5 \mathrm{eV}, 4.77 \mathrm{ev}$, and $4.31 \mathrm{eV}$ for diamond, silicon and germanium, respectively, obtained from the Penn expression 
for $\epsilon_{1}(0), 17$ because of the presence of the $\frac{2}{3}$ in the expression derived in this thesis.

The value of $11.2 \mathrm{eV}$ for $E_{g}$ for diamond agrees fairly well with Harrison's value of $10.8 \mathrm{eV}$ for the covalent energy of diamond, ${ }^{18}$ although he has taken a bond-orbital model approach to the calculation of the static dielectric constant. Harrison's covalent energy values are $3.0 \mathrm{eV}$ and $2.7 \mathrm{eV}$ for silicon and germanium, respectively, which are substantially smaller than the values obtained in this thesis. However, Harrison indicates that for group IV semiconductors with a sufficiently small gap a Penn-type model probably yields better results, but that his model is better for large gaps, ${ }^{19}$ because Harrison employs the tight-binding method.

Since the dielectric constant associated with a longitudinal disturbance is equal to the dielectric constant associated with a transverse disturbance in the limit of small $q, 11,13$ the energy gaps obtained from equations (53) and (54) can be used to generate theoretical curves for $\epsilon_{2}(E)$ by the use of equation (4I), where $E$ is the optical energy. For germanium, $E_{p}^{2}$ must be multiplied by Van Vechten's $D$ to take into account the effects of a core states on $\epsilon_{2}(E)$. Figures (3), (4), and (5) compare the theoretical curves from equation (41) with experimental results. To illustrate the dependence of the theoretical result on the value of $E_{g}$, these figures include an additional curve for an arbitrarily selected $E_{g}$. The experimental results for silicon and germanium are those of Phillip and Ehrenreich, ${ }^{20}$ while the experimental results for diamond are those of Phillip and Taft. ${ }^{21}$ The theoretical curves for $E_{g}$ calculated from $\epsilon_{1}(0)$ agree fairly well with the 
experimental results, but do not exhibit the structure of the experimental curves. This is because all interactions have been ignored, with the exception of the interactions of the electron with the electromagnetic field and the electron gas through the self-consistent-field approximation. ${ }^{11}$ From their detailed calculations Heine and Jones 22 have identified the higher energy peak of $\epsilon_{2}(E)$ with $E_{g}$, which is consistent with the results presented here.

The theoretical curves for $\epsilon_{1}(E)$ are obtained from the KramersKronig relation involving $\epsilon_{2}(E)$. The theoretical values of $E_{g}$ for diamond, silicon and germanium are substituted into equation (46). For $E<E_{g}$, the integral is evaluated numerically; for $E_{g}<E<4 E_{p}\left(1+\Delta^{2}\right)^{\frac{2}{2}}$, the integral is performed exactly. In figures (6), (7), and (8), the theoretical curves are shown so that a comparison can be drawn between them and the experimental curves. In all cases, the theoretical values of $\epsilon_{1}(E)$ are far too low except at low energies, but the theoretical curves do exhibit some qualitative agreement with the experimental curves. The following points should be made. The Penn model has been used so $\epsilon_{2}(E)$ is zero for $E \leq E_{g}$. The theoretical values for $\epsilon_{2}(E)$ are greater than the experimental values just above $E_{g}$ but are less that the experimental values in the high energy tails. In addition, the explicit band structure for the crystals has not been included. These points account for the quantitative departures of the theoretical curves from the experimental curves for $\epsilon_{1}(E)$ and for the lack of structure in the theoretical curves for $\epsilon_{1}(E)$ because $\epsilon_{1}(E)$ is obtained from $\epsilon_{2}(E)$ through the use of the Kramers-Kronig relation. 


\section{CONCLUDING REMARKS}

Improved expressions for the real and imaginary parts of the dielectric constant of semiconductors in the long wavelength limit have been obtained through the use of the Penn model. Phillips' theory of ionicity ${ }^{9}$ is based on Penn's expression for $\epsilon_{1}(0)$ rather than the improved expression given by equation (40). Before the implications of the improved expression for $\epsilon_{1}(0)$ on Phillips' theory of ionicity are discussed, a brief summary of Phillips' theory is in order.

In his theory, Phillips proposes to reduce the complicated bands in $K$ space to simple bonding and antibonding orbitals for binary $A^{N} B^{8-N}$ crystals where $N$ is the number of valence electrons for type $A$ atoms. The bonding and antibonding orbitals are $\varphi_{A}+\lambda \varphi_{B}$ and $\lambda \varphi_{A}-\varphi_{B}$, respectively, where $\varphi_{A}$ and $\varphi_{B}$ denote hybridized $s p^{3}$ orbitals suitable for tetrahedral coordination and $\lambda$ is a parameter whose value must be obtained variationally or by some other means. For all crystal structures considered, the diamond, zincblende, and rocksalt types have two atoms per unit cell while the wurzite type has four atoms per unit cell. Phillips indicates that all tensorial properties of wurzite crystals can be expressed in terms of those of zincblende crystals if interactions beyond those with nearest neighbors are neglected. Thus, Phillips contends that he is justified in constructing models for two atoms per unit cell. Phillips takes his potential to be a pseudopotential to avoid problems 
with cases where the cores of atoms A and B are not isoelectronic. With the coordinate origin chosen halfway between the two atoms in the unit cell, he finds that $E_{h}$ (the average energy gap between bonding and antibonding levels contributed by covalent forces) arises from the even part of the pseudopotential and C (the average energy gap between bonding and antibonding levels contributed by ionic forces) arises from the odd part of the pseudopotential. Using the structure factor appropriate to group IV.elements, he then determines the following relation, $E_{g}^{2}=E_{h}^{2}+c^{2} \quad . E_{g}$ is the average energy gap between bonding and antibonding levels. Harrison takes exception to Phillips' universal extension of the structure factor for group IV element: crystals to all other $A^{N} B^{8-N}$ crystals. ${ }^{19} E_{h}$ is equal to $E_{g}$ in the case of a purely covalent group IV crystal. Phillips argues that $E_{h}$ should depend only on bond length $l$. From Penn's expression for $\epsilon_{1}(0)$, Phillips calculates $E_{h}$ for diamond and silicon and finds that $E_{h} \propto l^{-2.5}$. From $E_{h}$ for silicon and the dependence on bond length, he calculates $E_{h}$ for other tetrahedrally coordinated $A^{N} B^{8-N}$ crystals. Penn's expression for $\epsilon_{1}(0)$ is modified by multiplying each value of $\omega_{p}^{2}$ by a correction factor (Van Vechten's $D^{10}$ ) to account for the effect of d-state electrons in other crystals. $E_{g}$ is calculated from the modified Penn expression and the observed dielectric constants. Knowing $E_{h}$ and $E_{g}$, Phillips can calculate $C$. Phillips defines $f_{i}$ as the ionicity and $f_{c}$ as the covalent fraction, where $f_{i}=\frac{c^{2}}{E_{g}^{2}}$, $f_{c}=\frac{E_{h}^{2}}{E_{g}^{2}}$, and $f_{i}+f_{c}=1$.

Since our values for diamond and silicon calculated from equation (53) also yield $E_{h} \propto l^{-2.5}$, use was made of the following 
expression to calculate $E_{h}$ for other crystals: $E_{h}=(3.92 \mathrm{eV})\left(\frac{l}{l_{s i}}\right)^{-2.5}$. Equation (54) was used to determine $E_{g}$ from experimental values of $\epsilon_{1}(0)$. Then, the values of $C$ and $f_{i}$ could be found. The values of $E_{h}, C, E_{g}$, and $f_{i}$ have been calculated for 34 crystals. Although the values of $E_{h}, C$, and $E_{g}$ are significantly different from Phillips and Van Vechten's values; in general, the ionicities differ only slightly from those of Phillips and Van Vechten (see Table I). The reason for this is that the ionicity as defined by Phillips is not sensitive to the constant which multiplies $\frac{E_{p}^{2}}{E_{g}^{2}}\left[1-\left(\frac{E_{g}}{4 E_{F}}\right)\right]$ in the expression for $\epsilon_{1}(0)$. In fact, if the term $\frac{E_{g}}{4 E_{F}}$ is neglected, the ionicity can be shown to be independent of this constant. Thus, Phillips' major result, the correlation of crystal structure with ionicity, is unaffected by the changes introduced through the improvements in Penn's calculations.

There are two possible applications of the improved expression for $\epsilon_{1}(0)$. The first would be to correct the various values of $b$ in Van Vechten's hypothesized expression ${ }^{10}$ for $C$ which is $c=b\left(\frac{z_{A} e^{2}}{r_{A}}-\frac{z_{B} e^{2}}{r_{B}}\right) e^{-k_{8} R}$, where $b$ is a dimensionless constant, $k_{s}$ is a linearized screening wave number equal to $\left(\frac{4 k_{F}}{\pi a_{0}}\right)^{\frac{1}{2}}$ ( $a_{\text {. }}$ is the Bohr radius), and $R=\frac{1}{2}\left(r_{A}+r_{B}\right) \cdot r_{A}$ and $r_{B}$ are covalent atomic radii $i^{17}$ for atoms $A$ and $B$, respectively. Since the improved expression for $\epsilon_{1}(0)$ resulted in values of $C$ significantly different from those of Phillips and Van Vechten, the corresponding values of $b$ will differ from Van Vechten's values. The success of Van Vechten's hypothesis is judged by how nearly constant b is for different crystals. His values of $C$ vary over a range of about a 
factor of ten, while his b values range over a factor of about 3 . It remains to be seen if $b$ is more nearly constant if the improved values of $C$ are used.

The second application would be to reinvestigate the effect of pressure on $\epsilon_{1}(0)$ by use of the improved expression for $\epsilon_{1}(0)$. The pressure variations of $\epsilon_{1}(0)$ depend on the functional dependence of $\epsilon_{1}(0)$ on $E_{g}$. The major change introduced in $\epsilon_{1}(0)$ from this work is the factor of $\frac{2}{3}$ discussed earlier. However, the functional form of $\epsilon_{1}(0)$ on $\Delta$ is also different. The differences become more important as the values of $\Delta$ become larger. Van Vechten has used Penn's expression to determine the effect of pressure on $\epsilon_{1}(0)$, but the agreement he obtained with experimental results is poor. Thus, this calculation should be repeated using the improved expression for $\epsilon_{1}(0)$. 
VII. APPENDIX

A. Calculation of $\epsilon_{2}(w)$ with $q$ Retained to second Order

In this appendix, equation (4I) presented above for $\epsilon_{2}(\omega)$ is derived when terms to second order in $q$ are retained in the delta function, $\delta\left(E_{\underline{k^{\prime}+q}}^{+}-E_{\underline{\sim}}^{-}-\hbar \omega\right)$. To perform the derivation, equation (39) is used as a starting point with the exception that $q$ has not been set equal to zero in the delta function.

$$
\epsilon_{2}(E)=\lim _{q \rightarrow 0} \frac{4 \pi^{2} e^{2}}{k_{F}^{2} \Omega} \sum_{k\left(\begin{array}{c}
1_{s} t \\
B . z_{.}
\end{array}\right)} \frac{z^{2} \Delta^{2}}{4\left[x^{2}+\Delta^{2}\right]^{2}} \delta\left(E_{\underline{k^{\prime}+q}}^{+}-E_{\underline{k}}^{-}-E\right),
$$

where $E=\hbar \omega$. To second order in $q$, the energy difference is

$$
\begin{aligned}
E_{\underline{k}^{\prime}+q}^{+}-E_{\underline{z}}^{-} & \cong E_{F}\left\{4\left(x^{2}+\Delta^{2}\right)^{\frac{1}{2}}-2 x\left[1+\left(x^{2}+\Delta^{2}\right)^{-\frac{1}{2}}\right] \eta_{z}\right. \\
& \left.+\eta^{2} z^{2}\left(x^{2}+\Delta^{2}\right)^{-1 / 2}+\eta^{2}\right\}
\end{aligned}
$$

where $\eta=q_{k_{F}}$ and $\quad x=1-\frac{k}{k_{F}}$.

The sum on $k$ in the first Brillouin zone is converted into an integral. The expression for $\epsilon_{2}(E)$ then becomes

$$
\begin{aligned}
& \epsilon_{2}(E) \cong \lim _{q \rightarrow 0} \frac{e^{2} \Delta^{2} k_{F}}{2} \int_{-1}^{1} \int_{0}^{1} \frac{(1-x)^{2} z^{2} d z d x}{\left[x^{2}+\Delta^{2}\right]^{2}} \delta\left\{E _ { F } \left[4\left(x^{2}+\Delta^{2}\right)^{\frac{1}{2}}\right.\right. \\
& \left.\left.-2 x\left(1+\left(x^{2}+\Delta^{2}\right)^{-\frac{1}{2}}\right) \eta z+\eta^{2} z^{2}\left(x^{2}+\Delta^{2}\right)^{-\frac{1}{2}}+\eta^{2}\right]-E\right\},
\end{aligned}
$$


35

where the lower limit on the $x$ integration has been set equal to 0 . Although this is an approximation, since $q$ is small but not equal to zero, it can be shown to introduce only terms of higher order in 9 than will be retained.

The delta function can be written as

$$
\delta[f(x)]=\frac{1}{\left|\frac{d f}{d x}\right|} \delta\left(x-x_{0}\right)
$$

where

$$
\begin{aligned}
& f(x)=E_{F}\left\{4\left(x^{2}+\Delta^{2}\right)^{1 / 2}-2 x\left[1+\left(x^{2}+\Delta^{2}\right)^{-1 / 2}\right] \eta z+\eta^{2} z^{2}\left(x^{2}+\Delta^{2}\right)^{-\frac{1}{2}}\right. \\
& \left.+\eta^{2}\right\}-E
\end{aligned}
$$

First, examine $f(x)$ and investigate the case where $E=E_{g}$. At $x=0, f(0)>0$ since the limit as $q \rightarrow 0$ has not been taken. For $x \neq 0$ and $\eta$ sufficiently small, it can be shown that $f(x)>0$ for $E=E_{g}$. Therefore, the delta function cannot be satisfied when $E=E_{g}$ and this point contributes nothing to $E_{2}(E)$. Next, $f(x)$ is set equal to zero to determine $x_{0}$,

$$
\begin{aligned}
& 4\left(x^{2}+\Delta^{2}\right)^{1 / 2}-2 x\left[1+\left(x^{2}+\Delta^{2}\right)^{-1 / 2}\right] \eta z+\eta^{2} z^{2}\left(x^{2}+\Delta^{2}\right)^{-1 / 2} \\
& +\eta^{2}-\frac{E}{E_{F}}=0 .
\end{aligned}
$$


If terms involving $\eta$ and $\eta^{2}$ are neglected, the solution is $x_{1}=\left[\left(\frac{E}{4 E_{F}}\right)^{2}-\Delta^{2}\right]^{1 / 2}$. Now, let $x_{0}=x_{1}+x$ where $x$ is small relative to $x_{1}$, and $x_{0}$ is the solution to (A.5). Binomial expansions are used and to second order in $\eta$ it can be shown that

$$
x_{0} \cong x_{1}+A(E) \eta z+B_{1}(E) \eta^{2} z^{2}+B_{2}(E) \eta^{2} \text {, }
$$

where

$$
\begin{aligned}
& A(E)=\frac{1}{2}\left[1+\frac{E}{4 E_{F}}\right], \\
& B_{1}(E)=\frac{1}{4}\left[\left(\frac{E}{4 E_{F}}\right)^{2}-\Delta^{2}\right]^{-\frac{1}{2}}\left[-1+\left(\frac{E}{4 E_{F}}\right)^{2}+\left(\frac{E}{4 E_{F}}\right)-\frac{1}{2} \Delta^{2}+\frac{1}{2} \Delta^{2}\left(\frac{4 E_{F}}{E}\right)^{2}\right]
\end{aligned}
$$

and

$$
B_{2}(E)=-\frac{1}{4}\left(\frac{E}{4 E_{F}}\right)\left[\left(\frac{E}{4 E_{F}}\right)^{2}-\Delta^{2}\right]^{-1 / 2}
$$

It can be shown that the expression for $x_{0}$ is valid for $E>E_{g}+\sigma\left(\eta^{n}\right)$ as long as $x_{0} \leq 1$, where $\sigma\left(\eta^{n}\right)$ is a term of order $n$ in $\eta$ and $0<n<2$. The region of invalidity vanishes in the limit as $\eta \rightarrow 0$

Finally, it can be shown that

$\delta\left\{E_{F}\left[4\left(x^{2}+\Delta^{2}\right)^{\frac{1}{2}}-2 x\left(1+\left(x^{2}+\Delta^{2}\right)^{-\frac{1}{2}}\right) \eta z+\eta^{2} z^{2}\left(x^{2}+\Delta^{2}\right)^{-\frac{1}{2}}+\eta^{2}\right]\right.$

$-E\} \cong \frac{\left(x^{2}+\Delta^{2}\right)^{\frac{3}{2}}}{E_{F}\left[4 x\left(x^{2}+\Delta^{2}\right)-2 x\left(\Delta^{2}+\left(x^{2}+\Delta^{2}\right)^{2}\right)-\eta^{2} z^{2} x\right]} \delta\left(x-x_{0}\right)$, 
where $x_{0}$ is given by equation (A.6). From equation (A.3) and (A.7),

$$
\epsilon_{2} \text { (E) can be shown to be }
$$

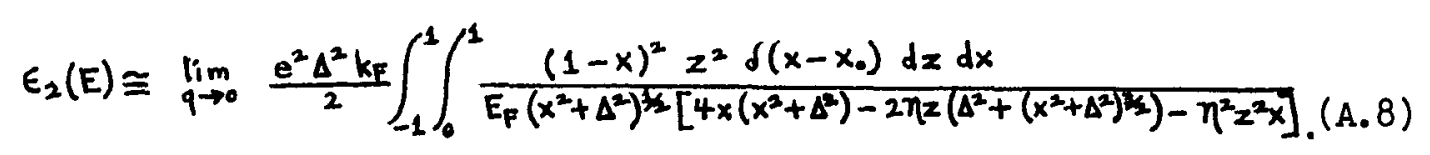

The expression finally obtained is

$$
\epsilon_{2}(E)=\frac{\pi}{2} \frac{E_{p}^{2}}{E^{3}} \frac{\left[E_{g}-\Delta\left(E^{2}-E_{g}^{2}\right)^{1 / 2}\right]^{2}}{\left(E^{2}-E_{g}^{2}\right)^{1 / 2}},
$$

for $E_{g}<E \leq 4 E_{F}\left(1+\Delta^{2}\right)^{3 / 2}$ and $\epsilon_{2}(E)=0$ for other values of $E$. Hence, a careful evaluation of the limit yields the same result as equation (4I) except directly at the energy gap, where the limit is not defined.

It can be shown that the Kramers-Kronig relation for $\epsilon_{1}(0)$ and $\epsilon_{2}(w)$ is satisfied through the use of equation (A.8). In addition, the agreement with the sum rule is the same as that obtained in equation (52).

B. Three-Band, Penn-Type Model

The purpose of this appendix is to estimate the effect of including more energy states on the $f$-sum rule. The approach is to employ a nearly-free-electron, three-band model that is isotropically extended to three dimensions (see figure 9). 
For the model, the gap at $2 k_{F}$ has been arbitrarily selected to be equal to the gap, $E_{g}$, at $k_{F}$. The wave functions and, accordingly, the energies have been arbitrarily picked to correspond to a nearly-free-electron model and are as follows:

for $0 \leq k<k_{F}$,

$$
\left|\psi_{\underline{x}, 1}(x)\right\rangle=\frac{e^{i \underline{k} \cdot r}}{\Omega^{1 / 2}}\left\{\frac{1-\beta_{\bar{k}} e^{-i 2 k_{F} \hat{k} \cdot r}}{\left[1+\left(\beta_{\underline{k}}\right)^{2}\right]^{1 / 2}}\right\},
$$

where $\left.\begin{array}{rl}\beta_{\bar{k}} & =\frac{\Delta}{\frac{k_{i}}{k_{F}}-1-\left[\left(\frac{k}{k_{F}}-1\right)^{2}+\Delta^{2}\right]^{1 / 2}} \text { and } \\ E_{\underline{\underline{k}, 1} 1}=E_{F}\left\{\frac{k^{2}}{k_{F}^{2}}-\frac{2 k}{k_{F}}+2-2\left[\left(\frac{k}{k_{F}}-1\right)^{2}+\Delta^{2}\right]^{1 / 2}\right\} ;\end{array}\right\}$

for $k_{F}<k<\frac{3}{2} k_{F}$,

$$
\left|\psi_{k, 2}^{l}(x)\right\rangle=\frac{e^{i k \cdot r}}{\Omega^{1 / 2}}\left\{\frac{1-\beta_{k}^{+} e^{-i 2 k_{F} \hat{k} \cdot c}}{\left[1+\left(\beta_{k}^{+}\right)^{2}\right]^{1 / 2}}\right\},
$$

where

$$
\begin{aligned}
\beta_{\underline{k}}^{+} & =\frac{\Delta}{\frac{k}{k_{F}}-1+\left[\left(\frac{k}{k_{F}}-1\right)^{2}+\Delta^{2}\right]^{3 / 2}} \quad \text { and } \\
E_{\underline{k}, 2}^{l}=E_{F}\left\{\frac{k^{2}}{k_{F}^{2}}-\frac{2 k}{k_{F}}+2+2\left[\left(\frac{k}{k_{F}}-1\right)^{2}+\Delta^{2}\right]^{1 / 2}\right\}_{j} & \}
\end{aligned}
$$


for $\frac{3}{2} k_{F}<k<2 k_{F}$,

$$
\left|\psi_{k, 2}^{\prime \prime}(x)\right\rangle=\frac{e^{i k \cdot r}}{\Omega^{1 / 2}}\left\{\frac{1-t_{k} e^{-i 4 k_{F} \hat{k} \cdot x}}{\left[1+\left(t_{k}\right)^{2}\right]^{1 / 2}}\right\},
$$

where $f_{k}=\frac{\frac{1}{2} \Delta}{\frac{k}{k_{p}}-2-\left[\left(\frac{k}{k_{F}}-2\right)^{2}+\frac{1}{4} \Delta^{2}\right]^{\frac{1}{2}}}$ and

$$
\left.E_{k, 2}^{\prime \prime}=E_{F}\left\{\frac{k^{2}}{k_{F}^{2}}-\frac{4 k}{k_{F}}+8-4\left[\left(\frac{k}{k_{F}}-2\right)^{2}+\frac{1}{4} \Delta^{2}\right]^{\frac{1}{2}}\right\}_{j}\right)
$$

and for $2 k_{F}<k<3 k_{F}$,

$$
\left|\psi_{k, 3}(x)\right\rangle=\frac{e^{i k \cdot x}}{\Omega^{1 / 2}}\left\{\frac{1-\gamma_{k}^{t} e^{-i+k_{k} \hat{k} \cdot x}}{\left[1+\left(\gamma_{k}^{t}\right)^{2}\right]^{1 / 2}}\right\},
$$

where $\quad f_{k}^{+}=\frac{\frac{1}{2} \Delta}{\frac{k}{k_{F}}-2+\left[\left(\frac{k}{k_{F}}-2\right)^{2}+\frac{1}{4} \Delta^{2}\right]^{\frac{1}{2}}}$

and

$$
E_{k, 3}=E_{F}\left\{\frac{k^{2}}{k_{F}^{2}}-\frac{4 k}{k_{F}}+8+4\left[\left(\frac{k}{k_{F}}-2\right)^{2}+\frac{1}{4} \Delta^{2}\right]^{\frac{1}{2}}\right\} .
$$

The subscripts 1,2 and 3 refer to the energy band while the superscripts $l$ and $u$ refer to the lower and upper half of band 2. If $E_{k, 2}^{l}$ and $E_{k, 2}^{u}$ are examined at $k=\frac{3}{2} k_{F}$, it is found that $E_{k, 2}^{l}-E_{k, 2}^{u}=3 \Delta^{2} E_{F}$. Since $\Delta$ is usually equal to 0.1 or less, the energies differ by $\frac{3}{4} \Delta E_{g}$ which is less than $0.1 E_{g}$. These energies are in fairly good agreement considering the fact that arbitrarily-selected, nearly-freeelectron wave functions and their corresponding energies were used. 
Before the matrix elements can be calculated for the Umklapp processes, an approximate expression for the matrix elements to first order in $q$ (that is, for small $q$ ) must be established. It can be shown that

$$
\left\langle\underset{k}{\underline{k}}\left|e^{-i q \cdot \mathfrak{r}}\right| \underline{k}+\underline{q}+\underline{G}\right\rangle \cong \frac{\frac{\hbar}{m}\left\langle\underline{k}\left|\mathfrak{l} \cdot \frac{\hbar}{i} \nabla\right| \underline{k}+G\right\rangle}{E_{k+G}-E_{\underline{k}}}
$$

for small $q$ - For positive frequencies, the expression for $\epsilon_{2}(w)$ can be written as

$$
\begin{aligned}
& \epsilon_{2}(\omega)=\lim _{q \rightarrow 0} \frac{4 \pi^{2} e^{2}}{q^{2} \Omega}\left\{\sum_{\left.k_{k,<}<k<k_{F}\right)}\left|\left\langle\psi_{k, 1}\left|e^{-i q \cdot \Sigma}\right| \psi_{k+q}^{l}-2 k_{F} \hat{k}, 2\right\rangle\right|^{2}\right. \\
& x \delta\left(E_{k+q-2 k, \hat{k}, 2}^{l}-E_{k, 1}-\hbar \omega\right)+\sum_{\left(0 \leq k<k_{2}\right)}\left|\left\langle\psi_{k, 1}\left|e^{-i q \cdot t}\right| \psi_{k+q-2 k, \hat{k}, 2}^{u}\right\rangle\right|^{2}
\end{aligned}
$$

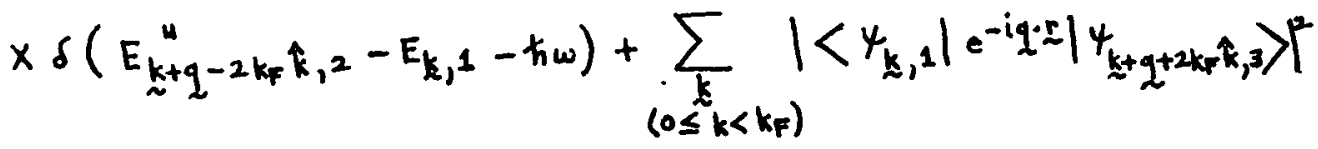

$$
\begin{aligned}
& x \delta\left(E_{k+q}+2 k_{f} k, 3-E_{k, 1}-\hbar \omega\right),
\end{aligned}
$$

where the wave functions and energies are given by equations (B.I) through (B.4). The matrix elements must now be calculated. The first matrix element has been calculated for the two-band model, but it is calculated here to prove the validity of our approximation for small 9 . 


$$
\left\langle\psi_{\underline{k}, 1}\left|e^{-i q \cdot i}\right| \psi_{k+q}^{l}-2 k_{p} \hat{k}, 2\right\rangle \cong-\frac{\pi z \Delta}{2\left[x^{2}+\Delta^{2}\right]},
$$

where $x=1-\frac{k}{k_{F}}, z=\cos \theta, \theta=$ angle between $k$ and $q$ and $\eta=\frac{q}{k_{F}}$. Thus, the approximate expression for the matrix element for small q yields the same result as the calculation performed before where direct algebraic manipulation was used. The other matrix elements are given by

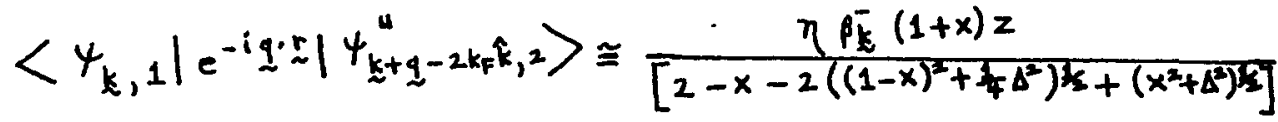

$$
\begin{aligned}
& x \frac{1}{\left[1+\left(\beta_{E}^{-}\right)^{2}\right]^{1 / 2}\left[1+\left(\gamma_{\underline{k}}^{-}-2 k_{F} k\right)^{2}\right]^{\frac{1}{2}}}
\end{aligned}
$$

and

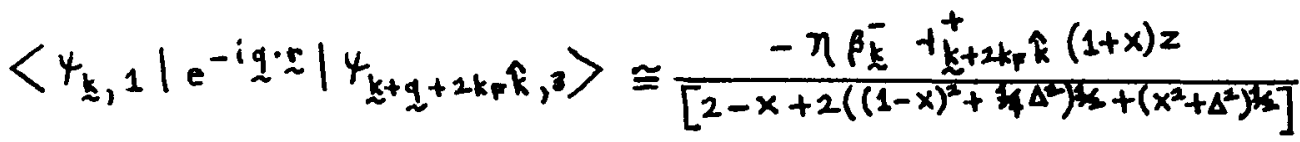

$$
\begin{aligned}
& x \frac{1}{\left[1+\left(\beta_{k}^{-}\right)^{2}\right]^{3 / 2}\left[1+\left(d_{k+2 k_{F} \hat{k}}^{+}\right)^{2}\right]^{\frac{1}{2}}} \text {. }
\end{aligned}
$$




$$
\beta_{\underline{L}}^{-}=\frac{\Delta}{-x-\left[x^{2}+\Delta^{2}\right]^{2}} \equiv \beta^{-}(x),
$$

$$
{\sqrt{k-2 k_{F} \hat{k}}}^{-}=\frac{\frac{1}{2} \Delta}{x-1-\left[(1-x)^{2}+\frac{1}{4} \Delta^{2}\right]^{3}} \equiv \mathcal{f}^{-}(x),
$$

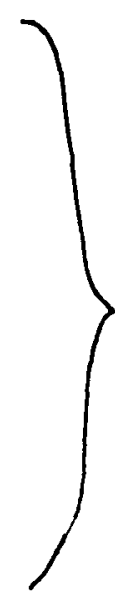

(B.10)

and $\quad f_{k+2 k_{F} \hat{k}}^{+}=\frac{\frac{1}{2} \Delta}{-x+1+\left[(1-x)^{2}+\frac{1}{4} \Delta^{2}\right]^{\frac{1}{2}}} \equiv f^{+}(x)$,

where $0 \leq x \leq 1$.

From equations (B.6) through (B.9), $\epsilon_{2}(E)$ can be shown to be

$$
\begin{aligned}
& \epsilon_{2}(E)=\frac{\pi}{2} E_{P}^{2}\left\{\Delta^{2} \int_{0}^{\frac{1}{2}} \frac{(1-x)^{2} d x}{4 E_{F}\left[x^{2}+\Delta^{2}\right]^{2}} \delta\left\{4 E_{F}\left(x^{2}+\Delta^{2}\right)^{\frac{1}{2}}-E\right\}\right. \\
& +\int_{\frac{1}{2}}^{1} \frac{\left(\beta^{-}(x)\right)^{2}\left(1-x^{2}\right)^{2} d x \delta\left\{2 E_{F}\left[2-x-2\left[(1-x)^{2}+\frac{1}{4} \Delta^{2}\right)^{\frac{1}{2}}+\left(x^{2}+\Delta^{2}\right)^{4}\right]-E\right\}}{E_{F}\left[2-x-2\left((1-x)^{2}+\frac{1}{4} \Delta^{2}\right)^{2 / 2}+\left(x^{2}+\Delta^{2}\right) t^{2}\right]^{2}\left[1+\left(\beta^{-2}(x)\right)^{2}\right]\left[1+\left(\gamma^{-}(x)\right)^{2}\right]}
\end{aligned}
$$

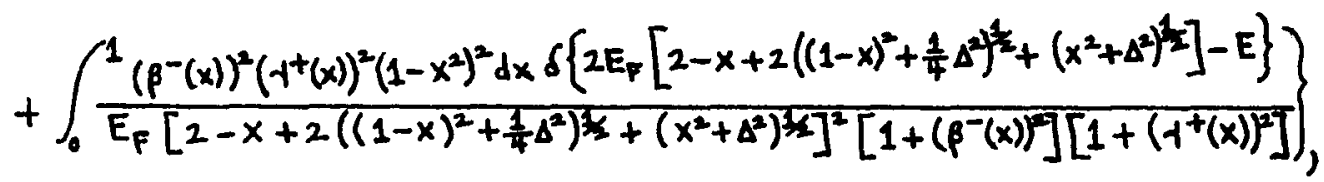

where $E=\hbar \omega$.

$$
\text { Now that an expression has been obtained for } G_{2}(E) \text {, the }
$$
$f$-sum rule can be investigated. It can be shown that 


$$
\begin{aligned}
& \frac{2}{\pi} \int_{0}^{\infty} E \epsilon_{2}(E) d E=E_{p}^{2}\left\{\left(\frac{1+3 \Delta^{2}}{2}\right)\left(\frac{1}{4}+\Delta^{2}\right)^{-\frac{1}{2}}-2 \Delta\right. \\
& +\Delta^{2} \log \left[\frac{\frac{1}{2}+\left(\frac{1}{4}+\Delta^{2}\right)^{\frac{1}{2}}}{\Delta}\right]+2 \int_{\frac{1}{2}}^{1}\left(\beta^{-(x)}\right)^{2}\left(1-x^{2}\right)^{2} d x \\
& x^{1 /[}\left[2-x-2\left((1-x)^{2}+\frac{1}{4} \Delta^{2}\right)^{\frac{1}{2}}+\left(x^{2}+\Delta^{2}\right)^{1}\right]\left[1+\left(\beta^{-}(x)\right)^{2}\right]\left[1+(r(x))^{2}\right] \\
& +2 \int_{0}^{1}\left(\beta^{-}(x)\right)^{2}\left(\gamma^{+}(x)\right)^{2}\left(1-x^{2}\right)^{2} d x \\
& \left.x^{1} /\left[2-x+2\left((1-x)^{2}+\frac{1}{4} \Delta^{2}\right)^{\frac{1}{2}}+\left(x^{2}+\Delta^{2}\right)^{\frac{1}{2}}\right]\left[1+\left(\beta^{-}(x)\right)^{2}\right]\left[1+\left(\lambda^{+}(x)\right)^{2}\right]\right\} .
\end{aligned}
$$

For $\Delta=0.1$, through the use of equation (B.10) the following result is obtained by evaluating the second and third integral numerically,

$$
\frac{2}{\pi} \int_{0}^{\infty} E \epsilon_{2}(E) d E=0.834 E_{p}^{2} \text {. }
$$

For all intents and purposes, this is the same result obtained with the two-band Penn model. Therefore, the inclusion of additional bands does not improve the situation as far as satisfying the $f$-sum rule is concerned. 


\section{ACKNOWLEDGEMENTS}

The research reported in this thesis was performed by the author as an employee of the National Aeronautics and Space Administration, Langley Research Center, Hampton, Virginia. The author wishes to express his gratitude to the National Aeronautics and Space Administration for endorsing this research. The author is grateful for the efforts of $\mathrm{Dr}$. Arden Sher expended in furthering the research. The author thanks Dr. Robert W. Shaw for suggesting this topic and for his helpful comments. The author is grateful to Dr. K. G. Petzinger and Dr. J. F. Soest for their helpful conversations and comments.

He also thanks Carl L. Fales for his worthwhile comments, William F. White for his suggestions relative to the computer programs used and William J. Debnam for his efforts. Finally, the author expresses his appreciation to his wife Vivian for her continued patience and encouragement without which this effort could never have been completed. 
IX. REFERENCES

1. John P. Walter and Marvin L. Cohen, Phys. Rev. B5, 3101 (1972);

S. J. Sramek and Marvin I. Cohen, Phys. Rev. B6, 3800 (1972).

2. D. R. Penn, Phys. Rev. 128, 2093 (1962).

3. J. Hubbard, Proc. Roy. Soc. A244, 199 (1958).

4. J. C. Phillips, Phys. Rev. 123, 420 (1961).

5. J. Callaway, Phys. Rev. 116, 1638 (1959).

6. G. Srinivasan, Phys. Rev. 178, 1244 (1969).

7. A. Bardasis and Daniel Hone, Phys. Rev. 153, 849 (1967).

8. Robert W. Shaw, Jr., Private Communication.

9. J. C. Phillips, The Reviews of Modern Physics, Vol. 42, No. 3, 317-356, July 1970.

10. J. A. Van Vechten, Phys. Rev. 182, 891 (1969).

11. H. Ehrenreich and M. H. Cohen, Phys. Rev. 115, 786 (1959).

12. P. Nozieres and D. Pines, Phys. Rev. 113, 1254 (1959).

13. David Pines, Elementary Excitation in Solids (W. A. Benfamin, Inc., New York, 1963), Chap. 4.

14. R. Brout and P. Carmuthers, Lectures on the Many-Electron Problem (Interscience Publishers, New York, 1963), Sec. 4.5.

15. J. M. Ziman, Principles of the Theory of Solids (Cambridge University Press, London, 1965), Sec. 5.6. The expression for $\epsilon(w, q)$ given by Ziman involves $e^{i q \cdot r}$ in the matrix element as a result of the way in which the perturbing potential and wave functions were selected. 
16. C. Kittel, Quantum Theory of Solids (John Wiley \& Sons, Inc., New York, London and Sydney, 1963), Chap. 9.

17. J. C. Phillips, Bonds and Bands in Semiconductors (Academic Press, New York and London, 1973), Chap. 2.

18. Walter A. Harrison, Phys. Rev. B8, 4487 (1973).

19. Walter A. Harrison, Private Communication.

20. H. R. Philipp and H. Ehrenreich, Phys. Rev. 129, 1550 (1963).

21. H. R. Philipp and E. A. Taft, Phys. Rev. 136, Al445 (1964).

22. V. Heine and R. O. Jones, J. Phys. C2, 719 (1969). 
X. LIST OF TABLES

1. Parameters of 34 Crystals 


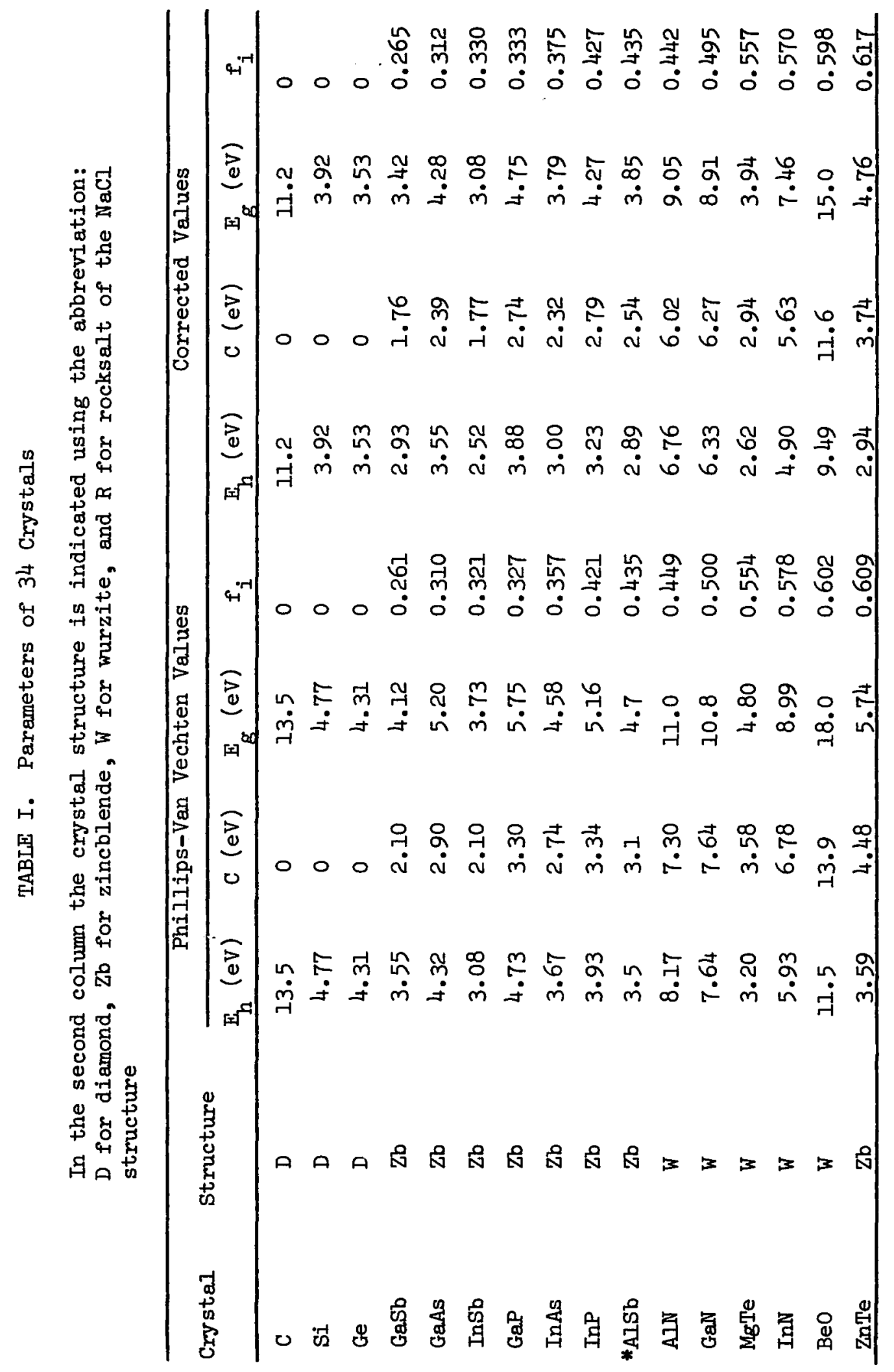




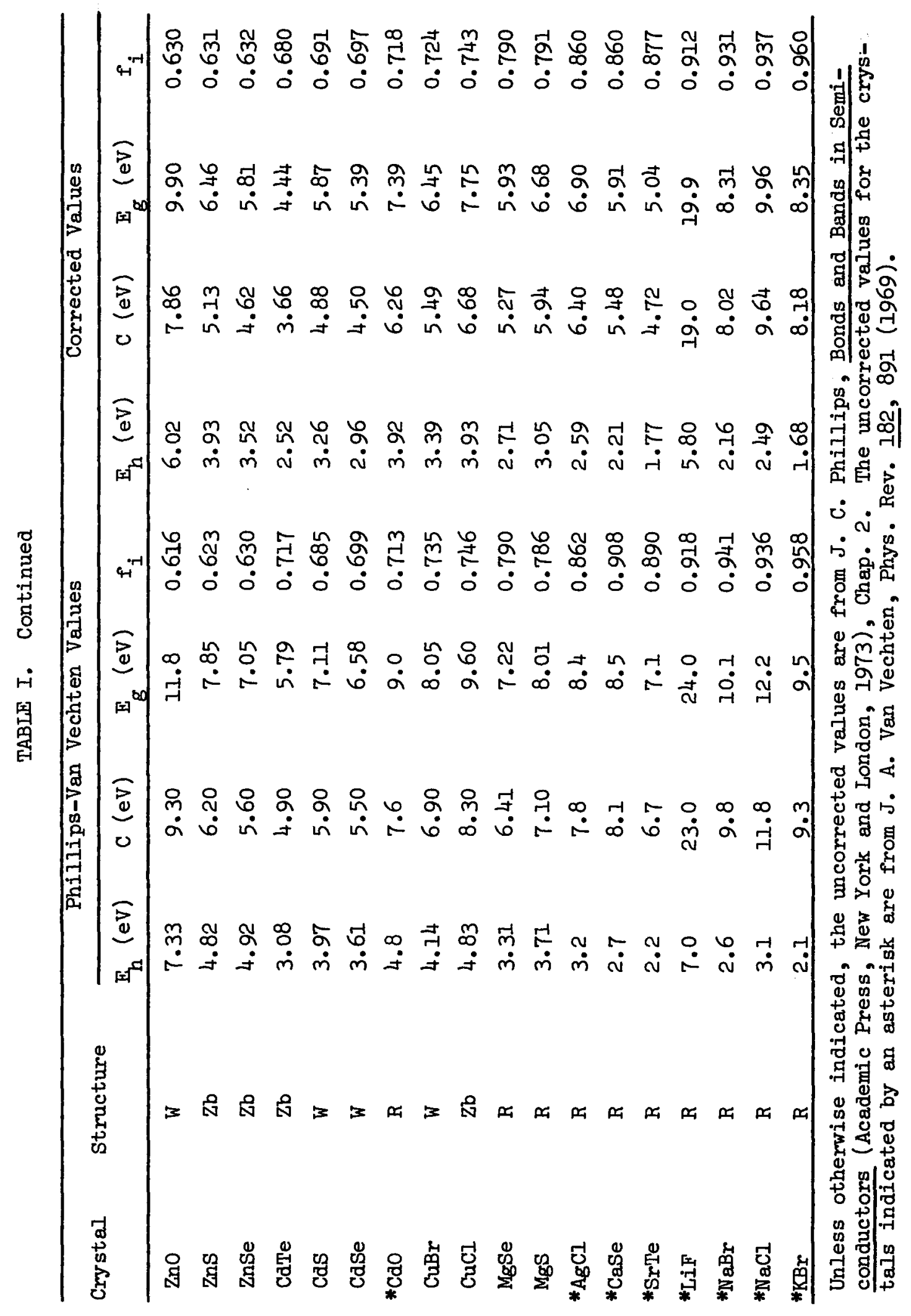




\section{LIST OF FIGURES}

1. Electron energy as a function of wave number for an isotropic, three-dimensional, nearly-free-electron model.

2. Construction of Srinivasan condition for the Penn model when $q$ is small.

3. The imaginary part of the dielectric constant in the long wavelength limit $(q \rightarrow 0)$ for diamond, as measured optically and as predicted by theory.

4. The imaginary part of the dielectric constant in the long wavelength limit $(q \rightarrow 0)$ for silicon, as measured optically and as predicted by theory.

5. The imaginary part of the dielectric constant in the long wavelength limit $(q \rightarrow 0)$ for germanium, as measured optically and as predicted by theory (with the Van Vechten correction for core d state effects included).

6. The real part of the dielectric constant in the long wavelength limit $(q \rightarrow 0)$ for diamond, as measured optically and as predicted by theory.

7. The real part of the dielectric constant in the long wavelength limit $(q \rightarrow 0)$ for silicon, as measured optically and as predicted by theory. 
8. The real part of the dielectric constant in the long wavelength limit $(q \rightarrow 0)$ for germanium, as measured optically and as predicted by theory (with the Van Vechten correction for core $d$ state effects included).

9. Electron energy as a function of wave number for a three-band, Penn-type model. 


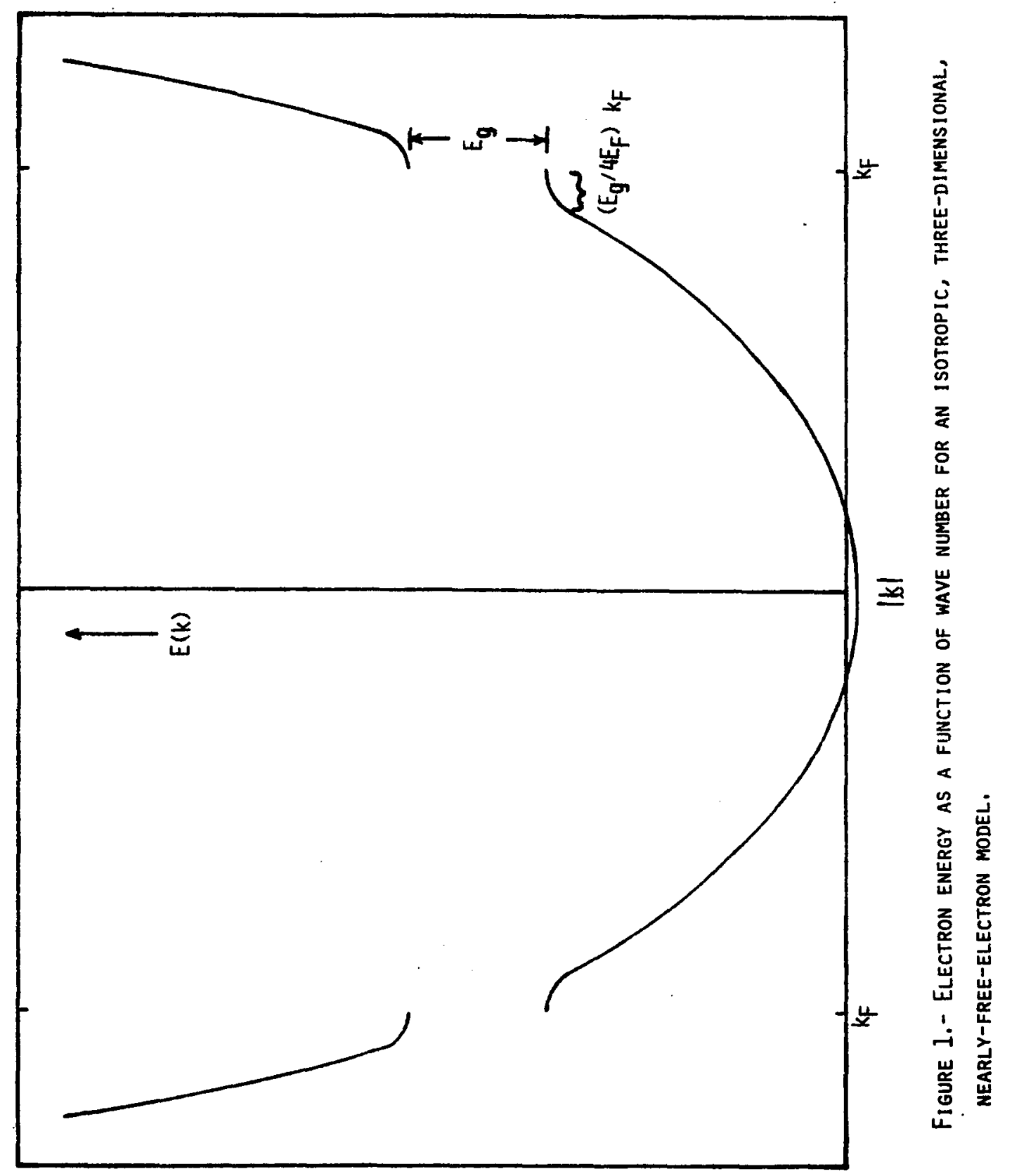




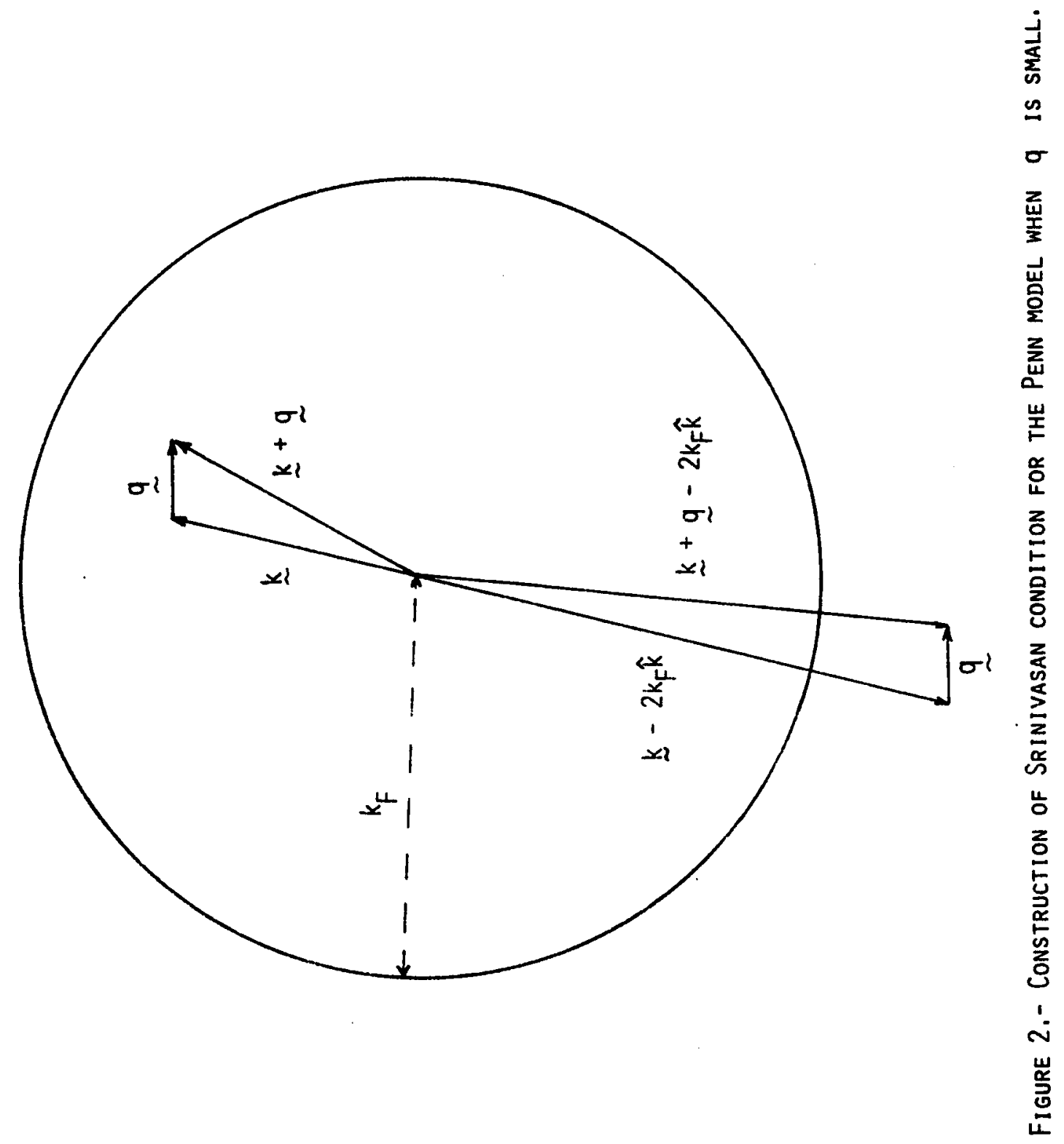




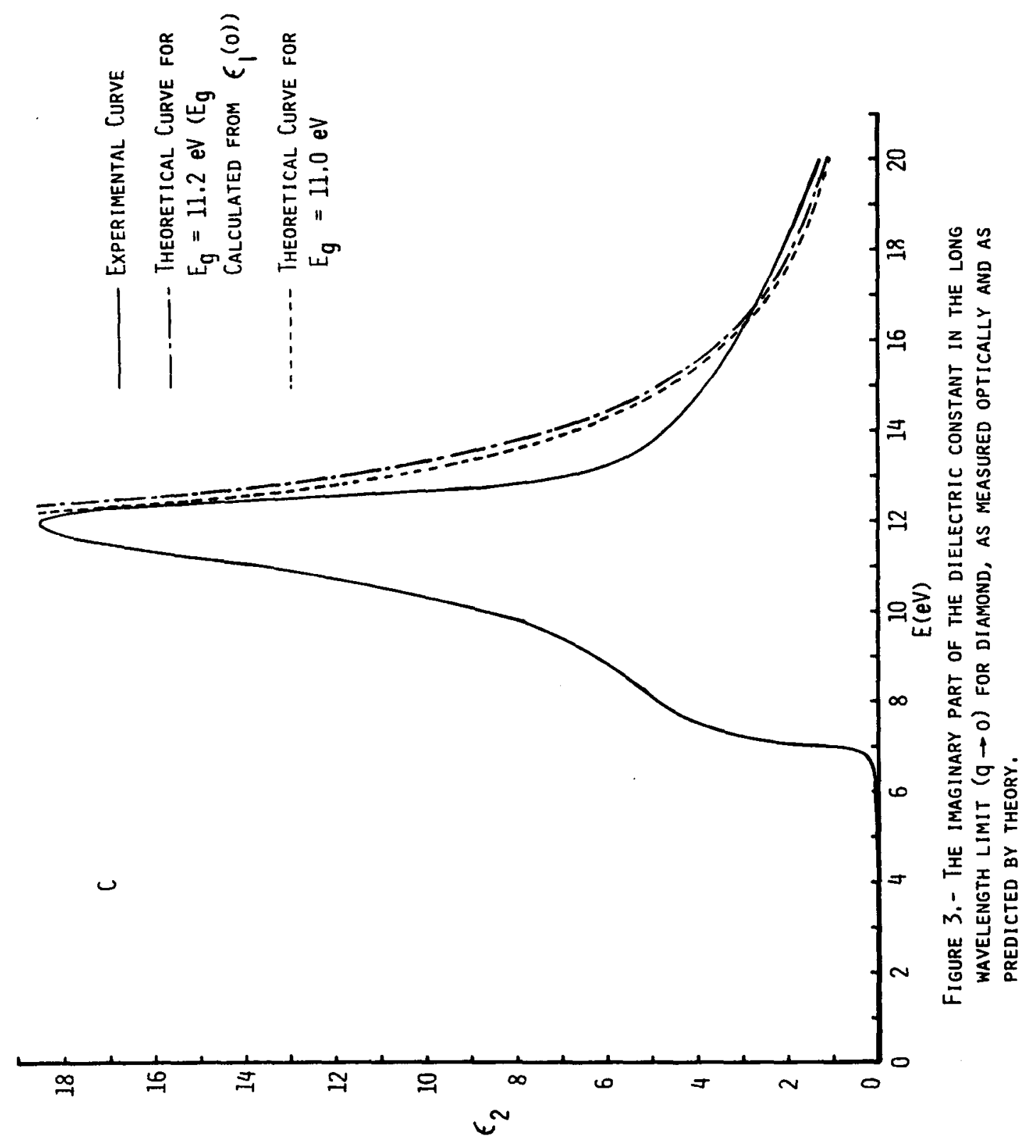




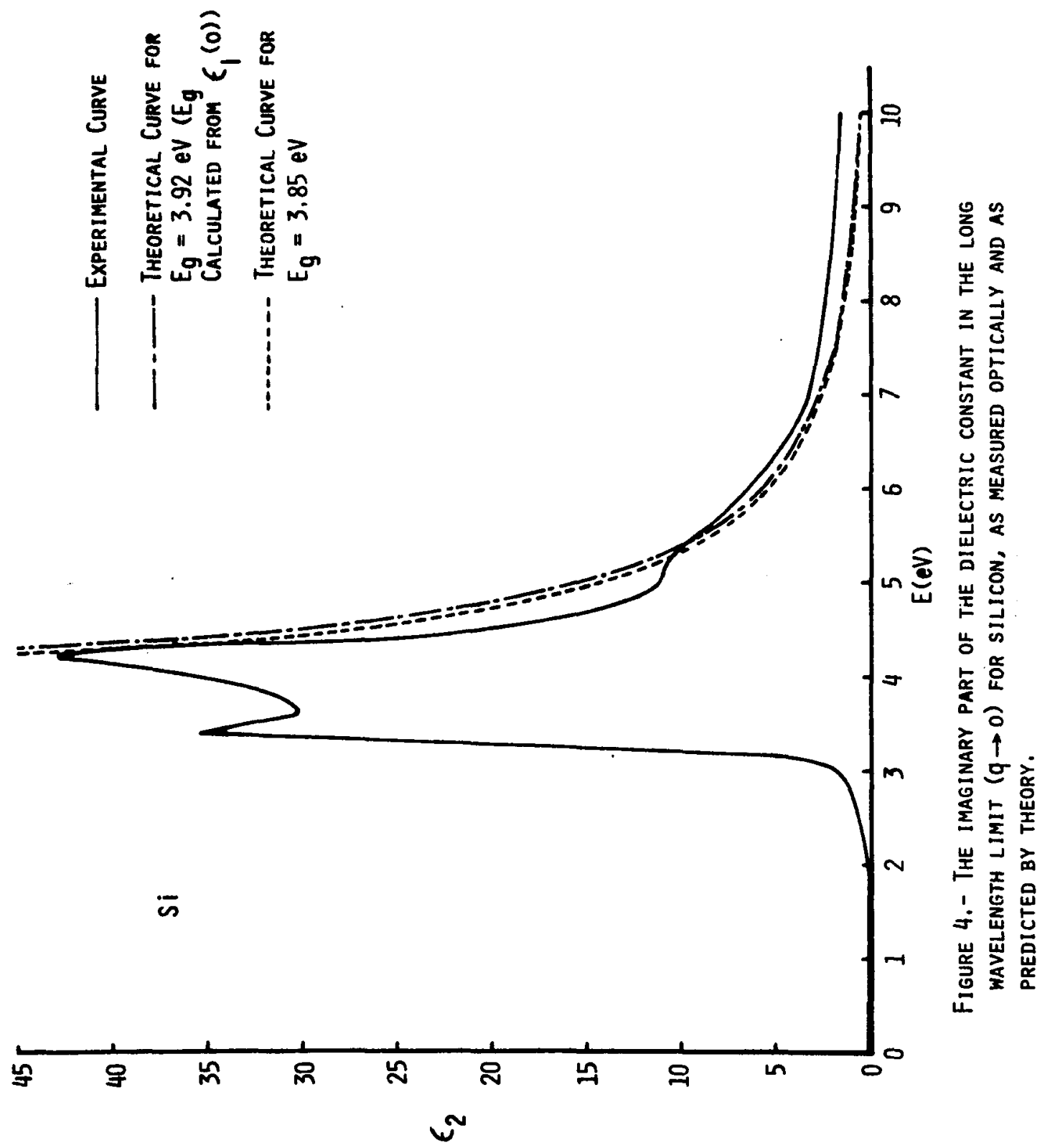




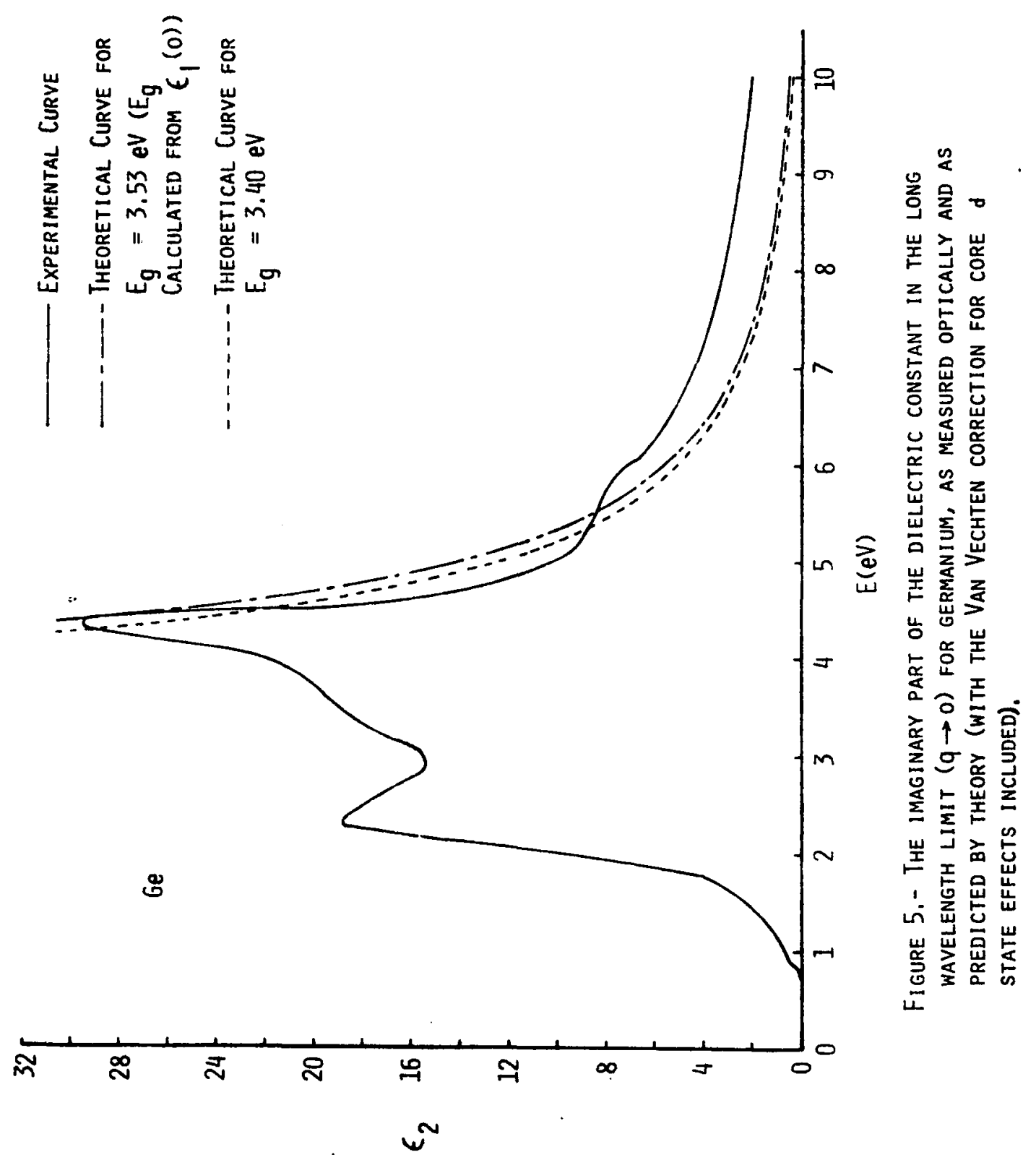




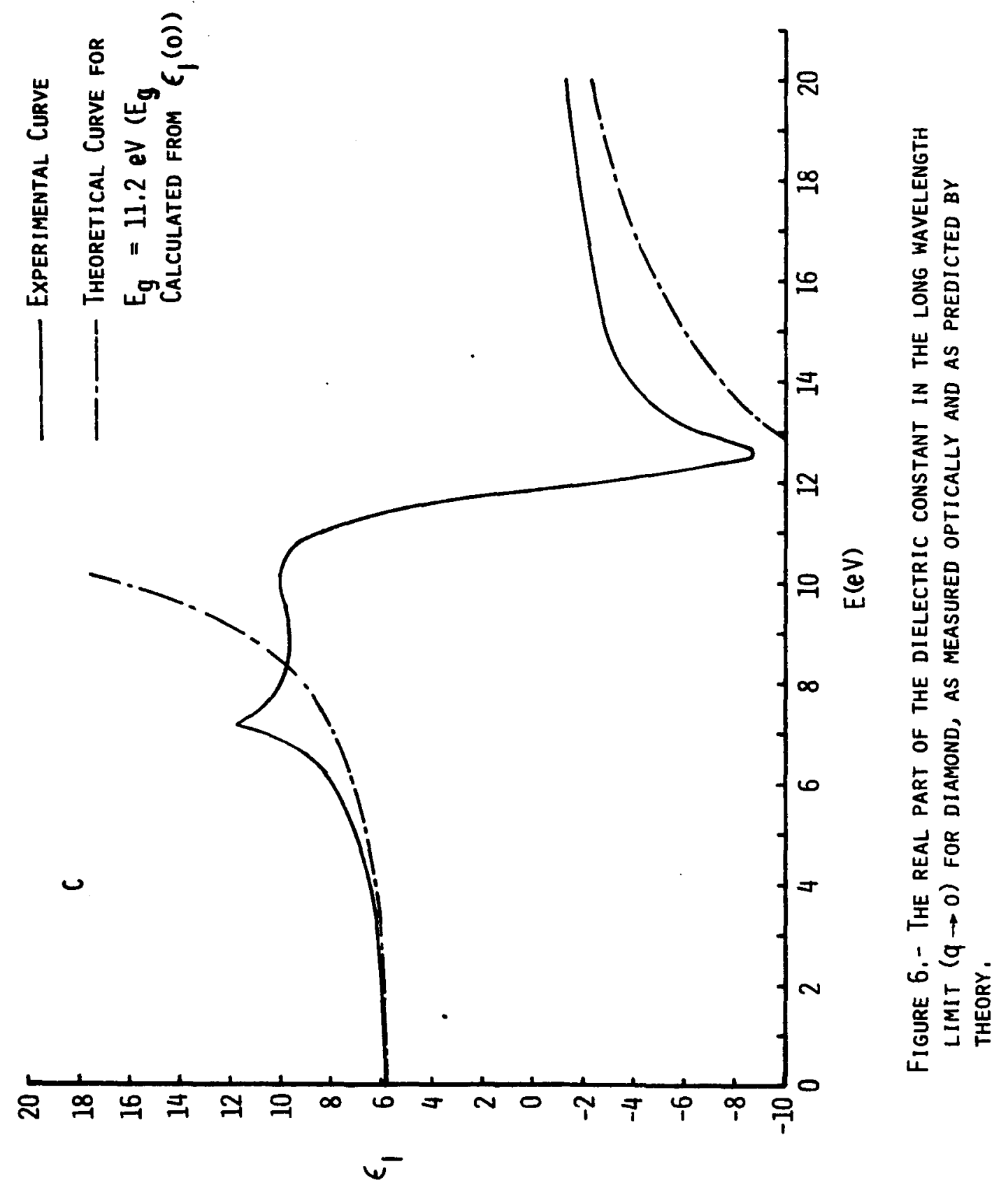




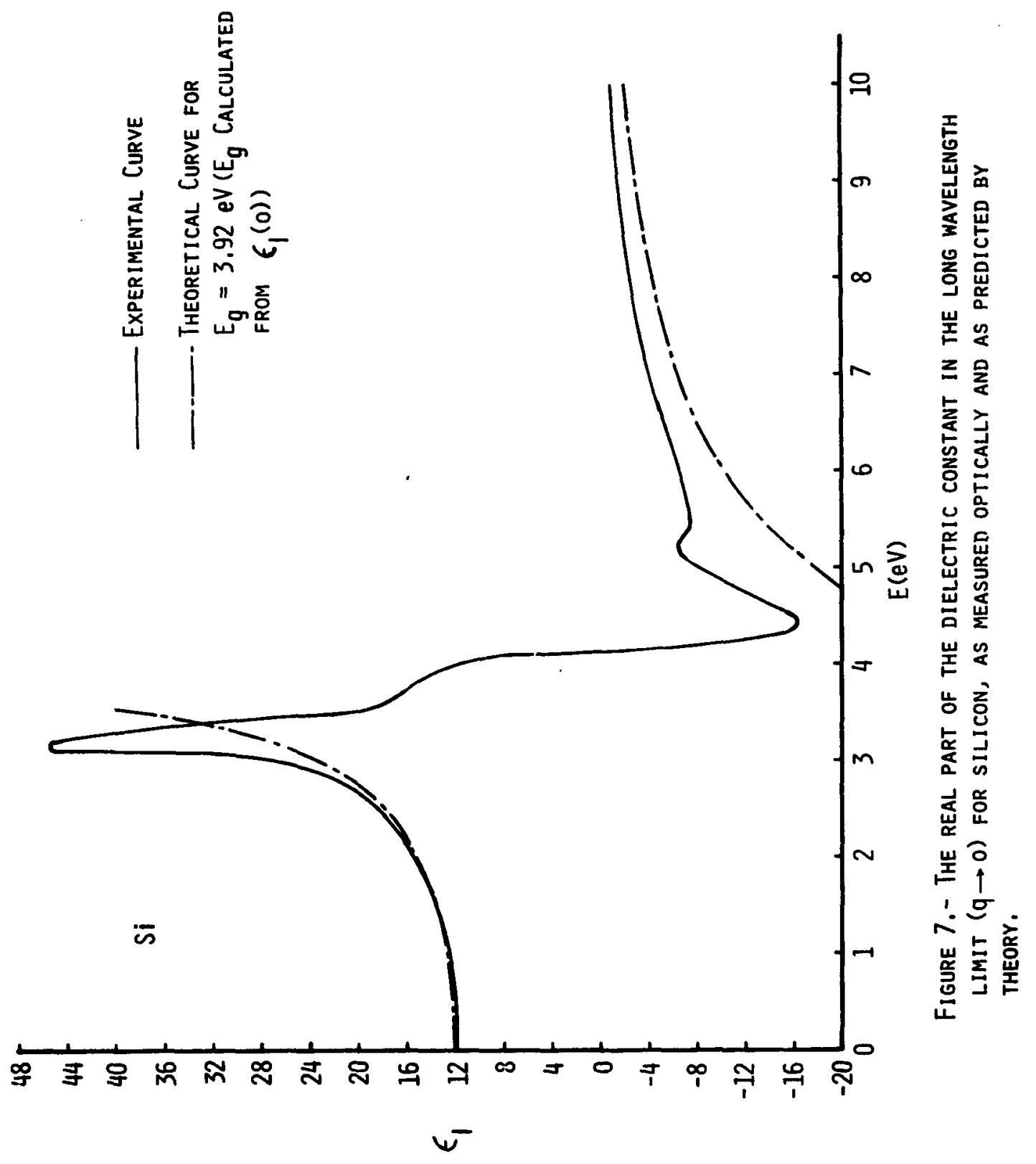



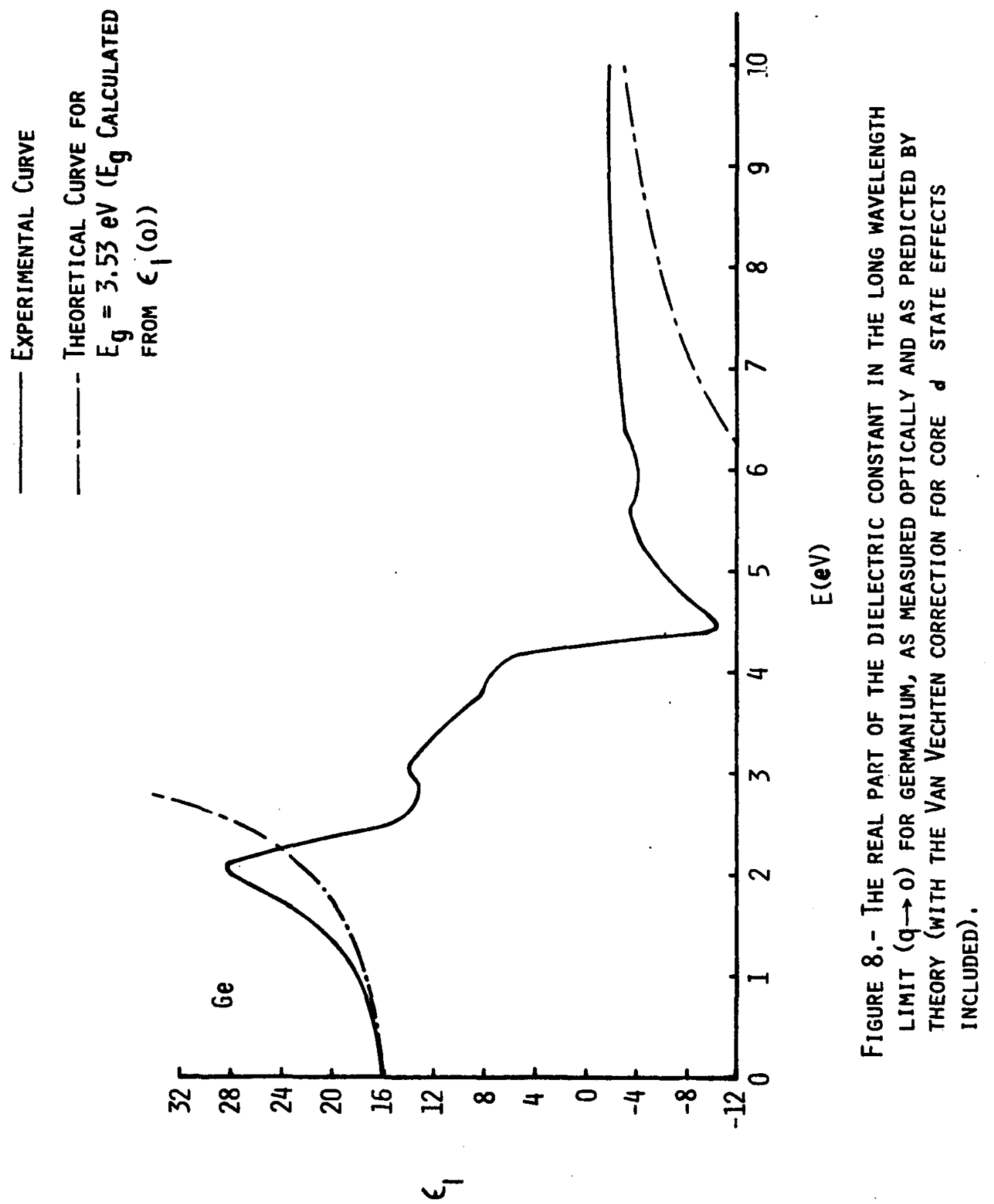


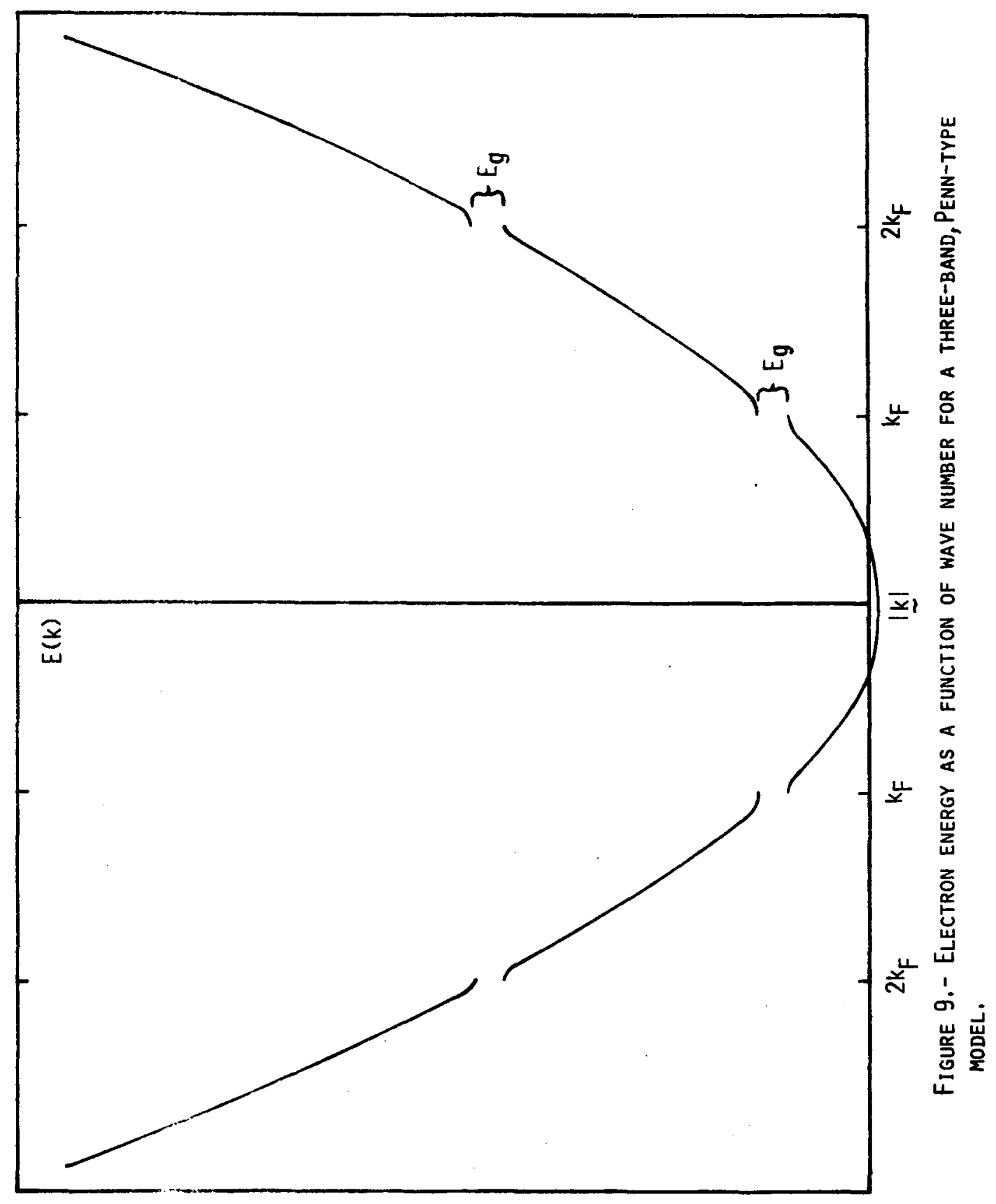

